\title{
The space of twisted cubics
}

\author{
Katharina Heinrich, Roy Skjelnes, and Jan Stevens
}

\begin{abstract}
We consider the Cohen-Macaulay compactification of the space of twisted cubics in $\mathbb{P}^{n}$. This compactification is the fine moduli scheme representing the functor of CM-curves with Hilbert polynomial $3 t+1$. We show that the moduli scheme of CM-curves in $\mathbb{P}^{3}$ is isomorphic to the twisted cubic component of the Hilbert scheme. We also describe the compactification for twisted cubics in $\mathbb{P}^{n}$.
\end{abstract}

Keywords. Hilbert schemes, twisted cubics, Cohen-Macaulay curves, Fitting ideals.

2020 Mathematics Subject Classification. 14C05, 14D22, 14H10, 14A20

\section{[Français]}

\section{L'espace des cubiques gauches}

Résumé. Nous considérons la compactification de Cohen-Macaulay de l'espace des cubiques gauches de $\mathbb{P}^{n}$. Cette compactification est l'espace de modules fin qui représente le foncteur des courbes $\mathrm{CM}$ avec polynôme de Hilbert $3 t+1$. Nous montrons que le schéma des modules des courbes $\mathrm{CM}$ dans $\mathbb{P}^{3}$ est isomorphe à la composante des cubiques gauches du schéma de Hilbert. Nous décrivons également la compactification pour les cubiques gauches de $\mathbb{P}^{n}$.

Received by the Editors on June 13, 2019, and in final form on February 18, 2021.

Accepted on March 5, 2021.

Katharina Heinrich

Department of Mathematics, KTH Royal Institute of Technology, SE 10044 Stockholm, Sweden.

e-mail:kchal@kth.se

Roy Skjelnes

Department of Mathematics, KTH Royal Institute of Technology, SE 10044 Stockholm, Sweden.

e-mail: skjelnes@kth.se

Jan Stevens

Department of Mathematical Sciences, Chalmers University of Technology and University of Gothenburg, SE 41296 Göteborg, Sweden.

e-mail: stevens@chalmers.se

(C) by the author(s) 


\section{Contents}

1. Introduction. . . . . . . . . . . . . . . . . . . . . . . . . . . . . . . . 2

2. Cohen-Macaulay curves . . . . . . . . . . . . . . . . . . . . . . . . . . . 3

3. Plain double points . . . . . . . . . . . . . . . . . . . . . . . . . . . . . . . 8

4. Singular sections of cubics . . . . . . . . . . . . . . . . . . . . . . . . . . 10

5. The space of twisted cubics. . . . . . . . . . . . . . . . . . . . . . . . . . . 12

A. The Hilbert scheme of twisted cubics. . . . . . . . . . . . . . . . . . . . . . 16

B. The moduli space of stable sheaves . . . . . . . . . . . . . . . . . . . . . . 20

References. . . . . . . . . . . . . . . . . . . . . . . . . . 21

\section{Introduction}

The main purpose of the present article is to provide a natural and functorial compactification of the space of twisted cubics in $\mathbb{P}^{3}$. The representing object is a smooth and irreducible projective scheme that is isomorphic to the twisted cubic component of the Hilbert scheme.

A twisted cubic is a smooth rational curve of degree three. The family of such curves in $\mathbb{P}^{3}$ is twelve dimensional. Finding a compactification of the parameter space with control over the degenerations is a fundamental question. An immediate answer is provided by the Hilbert scheme. In their celebrated work [PS85] Piene and Schlessinger described the Hilbert scheme Hilb $\mathbb{P}^{3}$ of space curves with Hilbert polynomial $3 t+1$. It consists of two smooth components. One component $\mathrm{H}$, the twisted cubic component, contains the twisted cubics as an open subset. The other component has as general member the union of a planar cubic and a point outside the plane.

An appealing feature of the Hilbert scheme is that it represents a functor, and thereby comes with universal defining properties. It gives a description of the families of curves including their degenerations. Its disadvantage is clear from the description above - it parametrizes also objects that are geometrically quite different from twisted cubics. Kontsevich's moduli space of stable maps $\overline{\mathscr{M}_{0,0}}\left(\mathbb{P}^{3}, 3\right)$ is another functorial compactification of the space of twisted cubics [Kon95, CK11]. The representing stack has been a marvelous tool for enumerative problems, but the geometry of this compactification is quite different from the twisted cubic component $\mathrm{H}$.

An interpolation between the Hilbert scheme and the Kontsevich moduli space was introduced by Hønsen [Høn05]. We refer to it as the space of CM-curves. A Cohen-Macaulay scheme $X$ of pure dimension one, together with a finite morphism $X \longrightarrow \mathbb{P}^{n}$ that generically is an immersion is a CM-curve (see Section 2). The functor of CM-curves in $\mathbb{P}^{n}$ with a fixed Hilbert polynomial is represented by a proper algebraic space ([Høn05], [Heil4]).

In the present article we use the space of CM-curves with Hilbert polynomial $3 t+1$ to compactify the space of twisted cubics. The most interesting case is that of curves in $\mathbb{P}^{3}$. Our main result (see Theorems 2.16 and 5.3) gives in particular a modular description of the twisted cubic component $\mathrm{H}$ :

Theorem. Let $\mathrm{CM}_{\mathbb{P}^{3}}^{3 t+1}$ be the space of $C M$-curves in $\mathbb{P}^{3}$ (over an algebraically closed field of arbitrary characteristic) with Hilbert polynomial $3 t+1$, and let $\mathrm{H} \subset \mathrm{Hilb}_{\mathbb{P}^{3}}^{3 t+1}$ denote the twisted cubic component of the Hilbert 
scheme. The space $\mathrm{CM}_{\mathbb{P}^{3}}^{3 t+1}$ is a smooth and irreducible projective scheme of dimension 12. We have an isomorphism

$$
\phi: \mathrm{CM}_{\mathbb{P}^{3}}^{3 t+1} \longrightarrow \mathrm{H} .
$$

The map $\phi$ yielding the isomorphism is obtained by giving the image of the finite map $i: X \longrightarrow \mathbb{P}^{3}$ the scheme structure determined by the $0^{\text {th }}$ Fitting ideal Fitt ${ }^{0}\left(i_{*} \mathscr{O}_{X}\right)$. This construction is discussed in detail in [Tei77]. It commutes with base change, but the resulting structure depends on the target of the morphism. Another important ingredient in our story is an explicit description of CM-curves in the plane. The space of CM-curves in $\mathbb{P}^{2}$ having Hilbert polynomial $3 t+1$ is identified with the space parametrising families of plane cubic curves, together with a section that passes through singular points of the family. We use a duality result by de Jong and van Straten relating Cohen-Macaulay ring extensions with the endomorphism ring of dual modules [dJvS90], which allows us to reconstruct the curve $X$ from its image and the singular section. For projective $n$-spaces with $n>3$ we identify a blow up of $\mathrm{CM}_{\mathbb{P}^{n}}^{3 t+1}$ with the twisted cubic component of Hilb $\mathbb{P}^{n}{ }^{n}$, based on the description of the Hilbert space in [CK11].

Other compactifications of the space of twisted cubics are known, see e.g. [EPS87, VX02, CK11]. The connections between different compactifications have been studied by [CK11] and by [Che08], the latter starting from the Kontsevich space. Freiermuth and Trautmann [FT04] studied (in characteristic 0) the moduli space of stable sheaves on $\mathbb{P}^{3}$ supported on cubic curves and found that it has two smooth components, one of which is isomorphic to the twisted cubic component $\mathrm{H}$ of the Hilbert scheme, see Appendix B. Their identification also uses Fitting ideals. Our results are valid in arbitrary characteristic; to this end we extend the results of [PS85] to also include characteristic 2 and 3, see Appendix A.

In general one cannot expect that a component of the space of CM-curves is isomorphic to a component of the corresponding Hilbert scheme - this already fails for $\mathrm{CM}_{\mathbb{P}^{n}}^{3 t+1}$ with $n>3$. Therefore the situation with Hilbert polynomial $3 t+1$ in $\mathbb{P}^{3}$ appears to be quite special. Only the case with Hilbert polynomial $2 t+2$ is similar and can be studied analogously. In characteristic zero it is known ([Har82, CCN11]) that the Hilbert scheme $\mathrm{Hilb}_{\mathbb{P}^{3}}^{2 t+2}$ consists of two smooth components. With the arguments in this article one can show that $\mathrm{CM}_{\mathbb{P}^{3}}^{2 t+2}$ is isomorphic to the component whose generic point corresponds to a pair of skew lines. In both cases only the simplest type of non-isomorphism occurs. This is no longer true for the case of quartic elliptic space curves. The irreducible component $\mathrm{H}$ of the Hilbert scheme Hilb $\mathbb{P}^{3}$ containing the points corresponding to these curves is explicitly known by the work of Avritzer and Vainsencher [VA92]; the Hilbert scheme consists of two components [Got08, LR11]. We expect that there exists a map from the component $\mathrm{H}$ to the space of $\mathrm{CM}$-curves. The picture is much more complicated for rational normal curves of degree 4, with Hilbert polynomial $4 t+1$. We hope that the study of the space of CM-curves can help in the study of the Hilbert scheme.

\section{Acknowledgments}

We are grateful for the comments of the anonymous referee that helped us to clarify certain arguments, and to improve the presentation of the article.

\section{Cohen-Macaulay curves}

In this section we define the space of CM-curves in general, and thereafter determine the dimension and prove smoothness when the Hilbert polynomial is $3 t+1$.

\subsection{The image of a finite map}

The schematic image of a quasi-compact morphism of schemes $f: X \longrightarrow Y$ is the closed subscheme of $Y$ determined by the kernel of the induced map $\mathscr{O}_{Y} \longrightarrow f_{*} \mathscr{O}_{X}$. When $f: X \longrightarrow Y$ is a finite morphism the ideal defining the schematic image is also given by the annihilator ideal $\operatorname{Ann}_{Y}\left(f_{*} \mathscr{O}_{X}\right)$. 


\subsection{Fitting ideals}

The $n^{\text {th }}$ Fitting ideal sheaf of a coherent $\mathscr{O}_{X}$-module $E$ is denoted by $\operatorname{Fitt}_{X}^{n}(E)$, or simply Fitt ${ }^{n}(E)$. We refer to [Nor76] or [Eis95] for definitions and their basic properties. We will be particularly interested in the $0^{\text {th }}$ Fitting ideal, inspired by the discussion by Teissier in [Tei77]. The ideal sheaf Fitt $_{X}^{0}(E)$ is obtained from the maximal minors of the matrix in a presentation of the module $E$.

\subsection{CM-curves}

We recall the notion of CM-curves as introduced by Hønsen [Høn05]. Let $\mathbb{P}^{n}$ denote the projective $n$-space over a fixed algebraically closed field $k$. A finite morphism $i: X \longrightarrow \mathbb{P}^{n}$ is a CM-curve if

(1) the scheme $X$ is Cohen-Macaulay and is of pure dimension one,

(2) the morphism $i$ is, apart from a finite set, an isomorphism onto its schematic image.

The Hilbert polynomial of a CM-curve $i: X \longrightarrow \mathbb{P}^{n}$ is the Hilbert polynomial of the coherent sheaf $i_{*} \mathscr{O}_{X}$ on $\mathbb{P}^{n}$.

\subsection{Functor of Cohen-Macaulay curves}

Let $p(t)=a t+b$ be a numerical polynomial. For a scheme $S$ we let $\mathrm{CM}_{\mathbb{P}^{n}}^{p(t)}(S)$ denote the set of isomorphism classes of pairs $(X, i)$ where $i: X \longrightarrow \mathbb{P}_{S}^{n}=\mathbb{P}^{n} \times S$ is a finite morphism of $S$-schemes, and where $X \longrightarrow S$ is flat, and for every geometric point in $S$ the fiber is a CM-curve with Hilbert polynomial $p(t)=a t+b$. Two pairs $(X, i)$ and $\left(X^{\prime}, i^{\prime}\right)$ are isomorphic if there exists an $S$-isomorphism $\alpha: X \longrightarrow X^{\prime}$ commuting with the maps $i$ and $i^{\prime}$. We have that $\mathrm{CM}_{\mathbb{P}^{n}}^{p(t)}$ is a contravariant functor from the category of locally Noetherian schemes, over our fixed field $k$, to Sets.

Theorem 2.5 (Hønsen, Heinrich). The functor $\mathrm{CM}_{\mathbb{P}^{n}}^{p(t)}$ of CM-curves in $\mathbb{P}^{n}$ having a fixed Hilbert polynomial $p(t)=a t+b$, is representable by a proper algebraic space.

Theorem 2.5 is proven more generally in [Høn05] for schemes defined over a fixed field of arbitrary characteristic, not necessarily algebraically closed. A different construction and proof is given in [Heil4] where it is shown that the functor is representable by a proper algebraic space of finite type over Spec $\mathbb{Z}$.

\subsection{Tangent space to $\mathrm{CM}$}

With $\mathbb{P}^{n}$ over a field $k$ and the polynomial $p(t)$ fixed, we write $\mathrm{CM}(R)$ for $\mathrm{CM}_{\mathbb{P}^{n}}^{p(t)}(\operatorname{Spec}(R))$ for any $k$ algebra $R$. Given a pair $\xi=(X, i)$ in $\mathrm{CM}(k)$ we define the functor $\mathrm{CM}_{\xi}[\mathrm{H} ø n 05, \S 3.4]$ on the category of local artinian $k$-algebras by

$$
\mathrm{CM}_{\xi}(R)=\left\{\left(X_{R}, i_{R}\right) \in \mathrm{CM}(R) \mid\left(X_{R}, i_{R}\right) \text { maps to } \xi \in \mathrm{CM}(k)\right\} \text {. }
$$

This is in fact a deformation functor.

For a morphism $i: X \longrightarrow Y$ Buchweitz [Buc81] considers six deformation functors, one of them being $\operatorname{Def}_{X / Y}$, the functor of deformations of $X$ over $Y$, where one deforms $X$ and the morphism $i$, but not the target $Y$. Its tangent space is $T_{X / Y}^{1}=\mathbb{E x t}_{\mathscr{O}_{X}}^{1}\left(\mathbb{L}_{X / Y}^{\bullet}, \mathscr{O}_{X}\right)$, where $\mathbb{L}_{X / Y}^{\bullet}$ is the cotangent complex [Ill72].

The functor $\mathrm{CM}_{\xi}$ is the functor Def $X / \mathbb{P}^{n}$ of deformations of $X$ over $\mathbb{P}^{n}$, the completion of the local ring of the point $(X, i) \in \mathrm{CM}(k)$ is the versal deformation of $X$ over $\mathbb{P}^{n}$ and the tangent space is $\mathrm{CM}_{\xi}(k[\varepsilon])$, where $\varepsilon^{2}=0$. In our specific situation we shall compute this tangent space by directly describing infinitesimal deformations of $(X, i)$. 


\subsection{Conductor}

Let $D=i(X)$ denote the schematic image of a CM-curve $i: X \longrightarrow \mathbb{P}^{n}$. We have the short exact sequence

$$
0 \longrightarrow \mathscr{O}_{D} \longrightarrow i_{*} \mathscr{O}_{X} \longrightarrow \mathscr{K}=i_{*} \mathscr{O}_{X} / \mathscr{O}_{D} \longrightarrow 0
$$

of sheaves on $D$. The target double point scheme [KLU92, p.204] of $i: X \longrightarrow \mathbb{P}^{n}$ is the closed subscheme $N_{X} \subseteq D$ defined by the ideal sheaf

$$
\operatorname{Ann}_{D}(\mathscr{K})=\operatorname{Ann}_{D}\left(i_{*} \mathscr{O}_{X} / \mathscr{O}_{D}\right) .
$$

This ideal sheaf equals the conductor of $\mathscr{O}_{D}$ in $i_{*} \mathscr{O}_{X}$. By definition the underlying set $\left|N_{X}\right|$ of points in $D$ is a finite set, and the open set $D \backslash N_{X}$ is isomorphic to $X \backslash i^{-1}\left(N_{X}\right)$.

\subsection{Twisted cubics}

The easiest example of a space of CM-curves is that of plane curves of degree $d$, which is a space that coincides with the Hilbert scheme. One of the easiest interesting, non-planar examples is the case of CMcurves with Hilbert polynomial $3 t+1$. In what follows we will assume that $\mathbb{P}^{n}$ is the projective $n$-space defined over an algebraically closed field.

Proposition 2.9. Let $i: X \longrightarrow \mathbb{P}^{n}$ be a CM-curve where $i$ is not a closed immersion. Assume that the Hilbert polynomial of $i_{*} \mathscr{O}_{X}$ is $3 t+1$. Then the image $D=i(X)$ is a plane cubic curve, and there is exactly one point $P \in D$ over which the morphism i fails to be an isomorphism.

Proof. The Hilbert polynomial of the skyscraper sheaf $\mathscr{K}=i_{*} \mathscr{O}_{X} / \mathscr{O}_{D}$ is a positive constant $\ell>0$; it is non-zero since by assumption the map $i$ is not a closed immersion. The Hilbert polynomial of $D$ is then $p(t)=3 t+1-\ell$. Hartshorne proved [Har94, Theorem 3.1] that the arithmetic genus $p_{a}=1-p(0)$ of the space curve $D \subseteq \mathbb{P}^{3}$ is bounded above by $p_{a} \leq \frac{1}{2}(d-1)(d-2)$, where $d$ is the degree of $D$, with equality if and only if $D$ is a plane curve. The same proof applies for $D \subset \mathbb{P}^{n}$, where $n \geq 3$ is arbitrary. It follows that $\ell=1$.

The support of $\mathscr{K}$ is then one point on $D$. The equality $p_{a}=\frac{1}{2}(d-1)(d-2)$ implies that the curve $D$ is planar.

Lemma 2.10. Let $i: X \longrightarrow \mathbb{P}^{n}$ be a CM-curve with Hilbert polynomial $3 t+1$. Then $H^{1}\left(X, i^{*} \mathscr{O}_{\mathbb{P}^{n}}(1)\right)=0$ and $\operatorname{dim}_{k} H^{0}\left(X, i^{*} \mathscr{O}_{\mathbb{P}^{n}}(1)\right)=4$.

Proof. Assume first that $i$ is a closed immersion. Define the index of speciality $e(X)$ of a Cohen-Macaulay curve $X \subset \mathbb{P}^{n}$ as the largest integer $e$ such that $H^{0}\left(X, \omega_{X}(-e)\right) \neq 0$, where $\omega_{X}$ is the dualizing sheaf. E. Schlesinger proves that $-2 \leq e(X) \leq \operatorname{deg}(X)-3$ [Sch99, Corollary 3.4]. This corollary applies as a (locally) Cohen-Macaulay curve is a quasi ACM subscheme of dimension 1 [Sch99, Corollary 2.7]. In our case $e(X) \leq 0$ and therefore $0=H^{0}\left(X, \omega_{X}(-1)\right)=H^{1}\left(X, \mathscr{O}_{X}(1)\right)$, where $\mathscr{O}_{X}(1)=i^{*} \mathscr{O}_{\mathbb{P}^{n}}(1)$. As the Hilbert polynomial is $3 t+1$ we then also have that $\operatorname{dim}_{k} H^{0}\left(X, \mathscr{O}_{X}(1)\right)=4$.

If the morphism $i$ is not a closed immersion then by Proposition 2.9 the image of $X$ is a planar cubic $D$. Tensoring the exact sequence (2.7.1) with $\mathscr{O}_{\mathbb{P}^{n}}(1)$ gives the exact sequence

$$
0 \longrightarrow \mathscr{O}_{D}(1) \longrightarrow i_{*} \mathscr{O}_{X}(1) \longrightarrow \mathscr{K} \longrightarrow 0
$$

of sheaves on $\mathbb{P}^{n}$. We have that $\operatorname{dim}_{k} H^{0}\left(D, \mathscr{O}_{D}(1)\right)=3$ and that $H^{1}\left(D, \mathscr{O}_{D}(1)\right)=0$ as $D$ is a planar cubic. It then follows from the exact sequence that $H^{1}\left(\mathbb{P}^{n}, i_{*} \mathscr{O}_{X}(1)\right)=0$ and that $\operatorname{dim}_{k} H^{0}\left(\mathbb{P}^{n}, i_{*} \mathscr{O}_{X}(1)\right)=4$. The projection formula gives that $i_{*} \mathscr{O}_{X}(1)=i_{*}\left(i^{*} \mathscr{O}_{\mathbb{P}^{n}}(1)\right)$, and since the morphism $i$ is finite the result follows.

Proposition 2.11. Let $i_{R}: X_{R} \longrightarrow \mathbb{P}_{R}^{n}$ be a CM-curve with Hilbert polynomial $3 t+1$. Assume that $R$ is a local ring. 
(1) We have that $H^{0}\left(X_{R}, i_{R}^{*} \mathscr{O}_{\mathbb{P}_{R}^{n}}(1)\right)$ is a free $R$-module of rank 4.

(2) Let us fix a basis of $H^{0}\left(X_{R}, i_{R}^{*} \mathscr{O}_{\mathbb{P}_{R}^{n}}(1)\right)$ and let $j_{R}: X_{R} \longrightarrow \mathbb{P}_{R}^{3}=\operatorname{Proj}\left(R\left[y_{0}, y_{1}, y_{2}, y_{3}\right]\right)$ be the induced morphism. Then $j_{R}$ is a closed immersion.

(3) There is a finite morphism $h_{R}: j_{R}\left(X_{R}\right) \longrightarrow \mathbb{P}_{R}^{n}$ induced by a linear map $R\left[x_{0}, \ldots, x_{n}\right] \longrightarrow R\left[y_{0}, \ldots, y_{3}\right]$, such that $i_{R}=h_{R} \circ j_{R}$.

(4) The pairs $\left(X_{R}, i_{R}\right)$ and $\left(j_{R}\left(X_{R}\right), h_{R}\right)$ are equal as points of the CM-functor.

Proof. Let $i: X \longrightarrow \mathbb{P}^{3}$ be the curve over the closed point of $\operatorname{Spec}(R)$. By Lemma 2.10 we have that $H^{r}\left(X, i^{*} \mathscr{O}_{\mathbb{P}^{n}}(1)\right)=0$ for $r>0$. Let $f_{R}: X_{R} \longrightarrow \operatorname{Spec}(R)$ be the structure map. It follows by cohomology and base change [Gro63, Corollaire 7.9.9] that $f_{R_{*}} i_{R}^{*} \mathscr{O}_{\mathbb{P}_{R}^{n}}(1)$ is free, and therefore by Lemma 2.10 free of rank 4 .

A choice of basis $\left(s_{0}, s_{1}, s_{2}, s_{3}\right)$ of global sections of $j_{R}^{*} \mathscr{O}(1)$ gives a morphism

$$
j_{R}: X_{R} \longrightarrow \mathbb{P}_{R}^{3}=\operatorname{Proj}\left(R\left[y_{0}, y_{1}, y_{2}, y_{3}\right]\right)
$$

such that $s_{i}=j_{R}^{*}\left(y_{i}\right), i=0, \ldots, 3$. The coordinates $\left(x_{0}: \cdots: x_{n}\right)$ on $\mathbb{P}_{R}^{n}$ give rise to global sections $i_{R}^{*}\left(x_{i}\right)$ of $i_{R}^{*} \mathscr{O}_{\mathbb{P}_{R}^{n}}(1)$, which can be expressed linearly in the basis $\left(s_{0}, s_{1}, s_{2}, s_{3}\right)$. This defines the map $h_{R}$.

To show that the finite morphism $j_{R}$ is a closed immersion it suffices to show that the induced map $j: X \longrightarrow \mathbb{P}^{3}$ over the closed point of $\operatorname{Spec}(R)$ is a closed immersion. If $j$ is not a closed immersion then we have by Proposition 2.9 that the image is contained in a plane in $\mathbb{P}^{3}$. This contradicts the fact that the global sections $s_{0}=j^{*}\left(y_{0}\right), \ldots, s_{3}=j^{*}\left(y_{3}\right)$ are linearly independent. It follows from the construction that the pairs $\left(j_{R}\left(X_{R}\right), h_{R}\right)$ and $\left(X_{R}, i_{R}\right)$ are isomorphic.

\subsection{Tangent space calculations}

The purpose of the rest of the present section is to prove that the CM-spaces with Hilbert polynomial $3 t+1$ are smooth. Smoothness is proven by showing that the dimension of the tangent space at the most special point equals the dimension of a general, smooth point. Later we will see that the most special situation is a projection of the curve $X \subset \mathbb{P}^{3}$ given by the graded ideal $I=\left(u^{2}, u y-x^{2}, x u\right) \subset k[x, y, u, w]$. Thus, for the moment we focus on this particular ideal. The canonical morphism $k[x, y, w] \longrightarrow k[x, y, u, w] / I$ of graded rings determines the finite morphism

$$
i_{1}: X \longrightarrow \mathbb{P}^{2}=\operatorname{Proj}(k[x, y, w]) .
$$

As such we have a CM-curve in $\mathbb{P}^{2}$ with Hilbert polynomial $3 t+1$. We will also view $\mathbb{P}^{2}$ as a closed subscheme in $\mathbb{P}^{3}$ given by the homogeneous ideal $(z) \subset k[x, y, z, w]$. The finite morphism (2.12.1) composed with the closed immersion gives a finite morphism

$$
i: X \rightarrow \mathbb{P}^{3},
$$

and thus a CM-curve in $\mathbb{P}^{3}$.

Lemma 2.13. Let $X \subseteq \mathbb{P}^{3}$ be the closed subscheme given by the graded ideal $I=\left(u^{2}, u y-x^{2}, x u\right) \subseteq k[x, y, u, w]$, and let $i: X \longrightarrow \mathbb{P}^{3}$ be the finite morphism (2.12.2). The completion of the local ring of the point $(X, i)$ in $\mathrm{CM}_{\mathbb{P}^{3}}^{3+1}$ is the power series ring $R=k\left[\left[a_{1}, \ldots, a_{8}, b_{9}, \ldots, b_{12}\right]\right]$. The universal family over $\operatorname{Spec}(R)$ is given by the pair $\left(X_{R}, i_{R}\right)$, where $X_{R}$ is given by the homogeneous ideal $J \subseteq R[x, y, u, w]$ generated by the maximal minors of

$$
\left(\begin{array}{ccc}
x+a_{2} w & a_{7} y+a_{6} w & u \\
y+a_{1} w & u+a_{5} x+a_{4} y+a_{3} w & x+a_{8} w
\end{array}\right)
$$

and the morphism $i_{R}: X_{R} \longrightarrow \mathbb{P}_{R}^{3}=\operatorname{Proj}(R[x, y, z, w])$ is determined by the linear map

$$
i_{R}^{*}(x, y, z, w)=\left(x, y, b_{9} x+b_{10} y+b_{11} w+b_{12} u, w\right) .
$$

Proof. We need to describe all deformations of the pair $(X, i)$. By Proposition 2.11 we may assume for a deformation $\left(X_{R}, i_{R}\right)$ over a local ring $R$ that the scheme $X_{R}$ is embedded in some $\mathbb{P}_{R}^{3}$ and the morphism $i_{R}$ 
is induced by a linear map. We start with infinitesimal deformations. The embedded deformations of the curve $X \subset \mathbb{P}^{3}=\operatorname{Proj}(k[x, y, u, w])$ are described in Lemma A.5, and are given by the maximal minors of

$$
\left(\begin{array}{ccc}
x+a_{2} w & a_{7} y+a_{6} w & u+a_{12} x+a_{11} y+a_{10} u+a_{9} w \\
y+a_{1} w & u+a_{5} x+a_{4} y+a_{3} w & x+a_{8} w
\end{array}\right)
$$

over the polynomial ring $k\left[a_{1}, \ldots, a_{12}\right]$. The morphism $i: X \longrightarrow \mathbb{P}^{3}$ is determined by the linear map $i^{*}(x, y, z, w)=(x, y, 0, w)$. This map is deformed by perturbing all possible entries. We need 16 variables $b_{1}, \ldots, b_{16}$ to perturb every component as a linear expression in $x, y, u, w$.

As we are allowed to perform coordinate transformations in the source of $i$, we can use the invertible map sending $x$ to the first component of the deformed map, $y$ to the second, $u$ to $u$ and $w$ to the fourth component. By performing these transformations the map simplifies to the linear map $i_{R}^{*}$ determined by

$$
i_{R}^{*}(x, y, z, w)=\left(x, y, b_{9} x+b_{10} y+b_{11} w+b_{12} u, w\right) .
$$

The matrix displayed above, describing the embedded deformations of the curve, then changes. But, after a linear change in the $a_{i}$ 's, depending on the $b_{j}$ 's we can take this matrix to be of the same form. The remaining available transformations can be used to make the $(1,3)$-entry of the matrix displayed into $u$. After another linear change of coordinates in the $a_{i}$ 's the infinitesimal deformations are given by the matrix (2.13.1) and the morphism $i_{R}$. These formulas define a deformation over the completion of the polynomial ring $k\left[a_{1}, \ldots, a_{8}, b_{9}, \ldots, b_{12}\right]$ in the ideal $\left(a_{1}, \ldots, a_{8}, b_{9}, \ldots b_{12}\right)$.

The next two lemmas will be used to describe the space of CM curves, with polynomial $3 t+1$, in the plane and in $\mathbb{P}^{n}$ with $n>3$ respectively.

Lemma 2.14. Let $X \subseteq \mathbb{P}^{3}$ be the closed subscheme given by the graded ideal $I=\left(u^{2}, u y-x^{2}, x u\right) \subseteq k[x, y, u, w]$, and let $i_{1}: X \longrightarrow \mathbb{P}^{2}$ be the morphism (2.12.1). The completion of the local ring of the point $\left(X, i_{1}\right)$ in $\mathrm{CM}_{\mathbb{P}^{2}}^{3 t+1}$ is given by the power series ring $R_{1}=k\left[\left[a_{1}, \ldots, a_{8}\right]\right]$. The universal family over $\operatorname{Spec}\left(R_{1}\right)$ is given by the pair $\left(X_{R_{1}}, i_{1}\right)$, where $X_{R_{1}}$ is given by the ideal $J \subseteq R_{1}[x, y, u, w]$ generated by the maximal minors of (2.13.1) and the morphism $i_{R_{1}}: X_{R_{1}} \longrightarrow \mathbb{P}_{R_{1}}^{2}$, is the one obtained from $i_{1}$.

Proof. We proceed as in the proof of Lemma 2.13. Now we perturb the map $i_{1}$ in all possible ways, and we can use coordinate transformations in the source to undo these perturbations.

Lemma 2.15. Let $X \subseteq \mathbb{P}^{3}$ be the closed subscheme given by the graded ideal $I=\left(u^{2}, u y-x^{2}, x u\right) \subseteq k[x, y, u, w]$ as in Lemma 2.14, but now let $i_{2}: X \longrightarrow \mathbb{P}^{n}=\operatorname{Proj}\left(k\left[x, y, w, z_{1}, \ldots, z_{n-2}\right]\right)(n>3)$ be the morphism determined by the linear map that sends $z_{i}$ to $0($ for $i=1, \ldots, n-2)$ and is the identity on $x, y$ and $w$.

The completion of the local ring of the point $\left(X, i_{2}\right)$ in $\mathrm{CM}_{\mathbb{P}^{n}}^{3 t+1}$ is given by the power series ring

$$
R_{2}=k\left[\left[a_{1}, \ldots, a_{8}, b_{1,1}, \ldots, b_{1,4}, b_{2,1}, \ldots, b_{n-2,4}\right]\right] .
$$

The universal family over $\operatorname{Spec}\left(R_{2}\right)$ is given by the pair $\left(X_{R_{2}}, j_{2}\right)$, where $X_{R_{2}}$ is given by the maximal minors of (2.13.1) and the morphism $i_{R_{2}}: X_{R_{2}} \longrightarrow \mathbb{P}^{n}$ is determined by the linear map that is the identity on $x, y$ and $w$, and that sends $\left(z_{1}, \ldots, z_{n-2}\right)$ to

$$
\left(b_{1,1} x+b_{1,2} y+b_{1,3} w+b_{1,4} u, \ldots, b_{n-2,1} x+b_{n-2,2} y+b_{n-2,3} w+b_{n-2,4} u\right) .
$$

Proof. Similar to the proof of Lemma 2.13 .

Proposition 2.16. The space $\mathrm{CM}_{\mathbb{P}^{3}}^{3 t+1}$ is smooth and irreducible of dimension 12.

Proof. Let $(X, i)$ be a $k$-point of $\mathrm{CM}=\mathrm{CM}_{\mathbb{P}^{3}}^{3 t+1}$ By Proposition 2.11 we may assume that $X$ is embedded in some $\mathbb{P}^{3}$ and that the morphism $i$ is the restriction of a rational map $h: \mathbb{P}^{3} \rightarrow \mathbb{P}^{3}$. In particular, $X$ itself is a curve on the twisted cubic component. Furthermore, the same holds for all infinitesimal deformations. 
If $i: X \longrightarrow \mathbb{P}^{3}$ is a closed immersion, then we may assume that $h$ is the identity and remains so under deformation. Consequently the infinitesimal deformations of $i: X \subset \mathbb{P}^{3}$ are the embedded deformations of $X$. Therefore the tangent space at the point $(X, i)$ is isomorphic to the tangent space to the twisted cubic component in the point $X$, which has dimension 12 [PS85, p. 766].

Assume now that $i: X \longrightarrow \mathbb{P}^{3}$ is not a closed immersion. By perturbing the coefficients defining the rational map $h$ we can deform the morphism $i: X \longrightarrow \mathbb{P}^{3}$ to a closed immersion. Thus any point on $\mathrm{CM}$ is the specialization of a twisted cubic in $\mathbb{P}^{3}$. It follows that the space $\mathrm{CM}$ is irreducible, and consequently to prove the proposition it suffices to show that the tangent space at any point has dimension 12 .

As the image $i(X)=D$ spans a plane, the map $h$ is the projection from a point $Q$. Because $i$ fails to be an isomorphism over exactly one point of $D$, the point $Q$ does not lie on two different tangent or secant lines. Considered as a point on the twisted cubic component, the curve $X \subset \mathbb{P}^{3}$ cannot be the most degenerate curve, which is a triple line given by the square of the ideal of a line [Har82, Section 1.b]. Such a curve has everywhere embedding dimension 3, so is not locally planar. Every other Cohen-Macaulay curve on the twisted cubic component degenerates to triple line on a quadric cone. For such a curve the center $Q$ of the projection does not lie on the tangent plane to the cone containing the line. All maps $i: X \longrightarrow \mathbb{P}^{3}$ with $X$ a triple line on a cone and $i$ generically an isomorphism are projectively equivalent, so it suffices to compute the tangent space in one specific example, for which we take the curve $(X, i)$ of Lemma 2.13. By specialization the dimension of the tangent space at any point is bounded from above by the dimension of the tangent space at this $(X, i)$, which is 12 .

\section{Corollary 2.17. For $n>3$, the space $\mathrm{CM}_{\mathbb{P}^{n}}^{3 t+1}$ is smooth and irreducible of dimension $4 n$.}

Proof. Let $i: X \longrightarrow \mathbb{P}^{n}$ be a CM-curve. If $i$ is a closed immersion it follows from Lemma 2.10 that the curve $X$ is contained in some $\mathbb{P}^{3}$, and from Proposition 2.11 that infinitesimal deformations of $i(X) \subset \mathbb{P}^{n}$ are

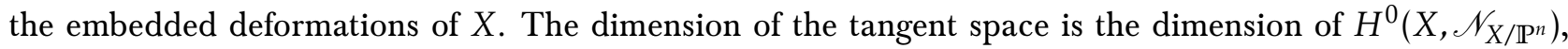
which can be computed as the sum of the dimensions of $H^{0}\left(X, \mathscr{N}_{X / \mathbb{P}^{3}}\right)$ and $H^{0}\left(X, \mathscr{N}_{\mathbb{P}^{3} / \mathbb{P}^{n}} \otimes \mathscr{O}_{X}\right)$, where $\mathscr{N}$ stands for the normal sheaf. The dimension is $12+4(n-3)=4 n$.

If $i$ is not a closed immersion then we have by Proposition 2.9 that the image is planar. Arguing as in the proof of Proposition 2.16 we see that the space CM is irreducible and that to conclude the proof it suffices to compute the dimension of the tangent space in the explicit example of Lemma 2.15. There the dimension is $4 n$.

Remark 2.18. We note that the space $\mathrm{CM}_{\mathbb{P}^{n}}^{3 t+1}$ is smooth and irreducible of dimension $4 n$, for all $n \geq 3$. The case $n=2$ will be described quite explicitly in Section 4 . In particular, the space $\mathrm{CM}_{\mathbb{P}^{2}}^{3 t+1}$ is smooth of dimension 8. This can also be shown using Lemma 2.14.

Remark 2.19. For CM-curves $(X, i)$ with Hilbert polynomial $2 t+2$ the analogue of Proposition 2.9 holds: if the map $i$ is not a closed immersion, then the image $D=i(X)$ is a singular plane conic, and there is exactly one point $P \in D$ over which the morphism $i$ fails to be an isomorphism. If $i: X \longrightarrow \mathbb{P}^{3}$ is a closed immersion, then the curve is not arithmetically Cohen-Macaulay, but one can still show that $\mathrm{CM}_{\mathbb{P}^{3}}^{2 t+2}$ is smooth and irreducible of dimension 8 .

\section{Plain double points}

In this section we focus on CM-curves where the non-isomorphism locus is the simplest possible. The following definition is motivated by Proposition 2.9.

Definition 3.1. Let $i: X \longrightarrow \mathbb{P}^{n}$ be a CM-curve, and let $D \subseteq \mathbb{P}^{n}$ denote its schematic image. A point $P$ in $D$ is a plain double point if the $\mathscr{O}_{D, P}$-module $\mathscr{K} \otimes_{\mathscr{O}_{D}} \mathscr{O}_{D, P}$ has length one, where $\mathscr{K}=i_{*} \mathscr{O}_{X} / \mathscr{O}_{D}$. 
Remark 3.2. The definition of a plain double point requires the double point locus of a map $i: X \longrightarrow \mathbb{P}^{n}$ to be as simple as possible. The point $P$ is always a singular point of the image, as a birational morphism onto a smooth curve is an isomorphism (apply this argument to a suitable affine neighbourhood of $P$ ). But the singularity might be of higher type. For instance, let $X$ be three lines in $\mathbb{P}^{3}$ meeting in one point, not lying in a plane, and let $i: X \longrightarrow \mathbb{P}^{2}$ be a general projection. Then the image $D=i(X)$ consists of three lines in the plane through one point $P$. The point $P \in D$ is a plain double point of the CM-curve $i: X \longrightarrow \mathbb{P}^{2}$. However the singularity $P \in D$ is a triple point and not a (planar) double point.

\subsection{The condition (R.C.)}

In the following the Ring Condition (R.C.) of De Jong and Van Straten [dJvS90] plays an important role. We recall the setup. Let $(R, \mathrm{~m})$ be a local ring, let $A$ be a local $R$-algebra, flat and Gorenstein over $R$. An $A$-module $M$ is Cohen-Macaulay over $R$ if it is flat and $\bar{M}=M \otimes_{R} R / \mathfrak{m}$ is Cohen-Macaulay. Write $\bar{A}$ for $A \otimes_{R} R / \mathrm{m}$. If furthermore the codimension $\operatorname{dim} \bar{A}-\operatorname{dim}_{\bar{A}} \bar{M}$ is zero we say that $M$ is maximally CohenMacaulay (MCM) over $R$. A fractional ideal is a finitely generated sub-module $M$ of the total fraction ring $Q A$ of $A$, containing a non-zero divisor. By [dJvS90, Propopsition 1.7] the duality functor $M \mapsto$ $\operatorname{Hom}_{A}(M, A)$ is an inclusion reversing involution on the category of fractional MCM's over $R$, commuting with specialization for MCM's. In this situation we have the following result [dJvS90, Propopsition 1.8].

Proposition 3.4. Let $A$ be an R-algebra, flat and Gorenstein over $R$, let $B$ be a fractional MCM A-module over $R$ and let $C=\operatorname{Hom}_{A}(B, A)$ be its dual module. Then $B$ is a ring (with ring structure induced from $\left.B \subset Q A\right)$ if and only if the natural inclusion map

$$
\operatorname{Hom}_{A}(C, C) \hookrightarrow \operatorname{Hom}_{A}(C, A)
$$

is an isomorphism.

A submodule $C$ of a ring $A$, that is an ideal, satisfies the Condition (R.C.) if the natural inclusion map (3.4.1) is an isomorphism.

Proposition 3.5. Let $\left(X_{R}, i_{R}\right) \in \mathrm{CM}_{\mathbb{P}^{2}}(\operatorname{Spec} R)$ with $R$ a local ring. Let $i: X \longrightarrow \mathbb{P}^{2}$ denote the induced curve over the closed point in $R$, and assume that $P \in \mathbb{P}^{2}$ is a plain double point. Let $\xi \in \mathbb{P}_{R}^{2}$ be the image of the point $P$ under the natural inclusion $\mathbb{P}^{2} \subseteq \mathbb{P}_{R}^{2}$. Then $\xi$ is a point on the schematic image $D_{R} \subseteq \mathbb{P}_{R}^{2}$ of $X_{R}$, and we have a presentation

$$
\oplus_{i=1}^{2} \mathscr{O}_{\mathbb{P}_{R}^{2}, \xi} \stackrel{\left(\begin{array}{ll}
g & f \\
s & t
\end{array}\right)}{\longrightarrow} \oplus_{i=1}^{2} \mathscr{O}_{\mathbb{P}_{R}^{2}, \xi} \longrightarrow i_{R *} \mathscr{O}_{X_{R}, \xi} \longrightarrow 0 .
$$

where $s$ and $t$ are elements in the maximal ideal of $\mathscr{O}_{\mathbb{P}_{R}^{2}, \xi}$ and $g$ and $f$ lie in the ideal $\mathrm{n}_{R}=(s, t)$. Furthermore, we have the equality of ideals $\mathfrak{n}_{R}=\operatorname{Ann}_{D_{R}}\left(i_{R *} \mathscr{O}_{X_{R}} / \mathscr{O}_{D_{R}}\right)$.

Proof. Let $\mathfrak{n}$ denote the maximal ideal of the local ring $A=\mathscr{O}_{D, P}$, and set $B=i_{*} \mathscr{O}_{X, P}$. It follows that $\mathfrak{n}$ equals the annihilator ideal $\operatorname{Ann}_{A}(B / A)$, which equals the conductor ideal $\{a \in A \mid a B \subseteq A\}$. The fact that $B$ is a ring gives by Proposition 3.4 that $B=\operatorname{Hom}_{A}(\mathfrak{n}, A)$ is isomorphic to $\operatorname{Hom}_{A}(\mathfrak{n}, \mathfrak{n})$.

We may assume that the plain double point $P$ is $(0: 0: 1)$, so the maximal ideal $n$ is the ideal $(x, y)$. As $P$ is a plain double point the $A$-module $B$ is generated by two elements, say 1 and $u$. The element $u$ corresponds to an endomorphism $\mu_{u}: \mathfrak{n} \longrightarrow \mathfrak{n}$. We get that $\mu_{u}(x)=-\bar{g}$ and $\mu_{u}(y)=-\bar{f}$ for some $\bar{f}$ and $\bar{g}$ in $\mathrm{n}$. In other words we have that $u x+\bar{g}=0$ and $u y+\bar{f}=0$. We get a surjective map $A \oplus A \longrightarrow B$ given by $\left(a_{1}, a_{2}\right) \mapsto a_{1}+a_{2} u$. The kernel contains the elements $(\bar{g}, x)$ and $(\bar{f}, y)$ and they in fact generate the kernel: the image of $(a, 1)$ is $a+u$, which is not zero as $u \notin A$. We have therefore the presentation

$$
\oplus_{i=1}^{2} A \stackrel{\left(\begin{array}{ll}
\bar{g} & \bar{f} \\
x & y
\end{array}\right)}{\longrightarrow} \oplus_{i=1}^{2} A \longrightarrow B \longrightarrow 0 .
$$


As $B$ is a Cohen-Macaulay $\mathscr{O}_{\mathbb{P}^{2}, P}$-module of codimension one, generated by 1 and $u$, it has a free resolution of length one. Therefore we have a presentation over $\mathscr{O}_{\mathbb{P}^{2}, P}$, obviously given by the same matrix where we use the same notation for elements in $\mathscr{O}_{\mathbb{P}^{2}, P}$ as for elements in $A$. The determinant $x \bar{f}-y \bar{g}$ of the matrix defines the scheme-theoretic image $i(X)=D$ locally around $P$. By assumption $X_{R} \longrightarrow \operatorname{Spec}(R)$ is flat and it follows that the presentation over $\mathscr{O}_{\mathbb{P}^{2}, P}$ can be lifted to a presentation over $\mathscr{O}_{\mathbb{P}_{R}^{2}, \xi}$, where $\xi$ is the image of $P$, see e.g. [Art76]. Thus, there are elements $s, t, g, f$ in $\mathscr{O}_{\mathbb{P}_{R}^{2}, \xi}$ that specialize to $x, y, \bar{g}, \bar{f}$ in $\mathscr{O}_{\mathbb{P}^{2}, P}$, respectively, giving us the exact sequence

$$
\oplus_{i=1}^{2} \mathscr{O}_{\mathbb{P}_{R}^{2}, \xi} \stackrel{\left(\begin{array}{ll}
g & f \\
s & t
\end{array}\right)}{\longrightarrow} \oplus_{i=1}^{2} \mathscr{O}_{\mathbb{P}_{R}^{2}, \xi} \longrightarrow i_{R *} \mathscr{O}_{X_{R}, \xi} \longrightarrow 0 .
$$

The ideal $I$ of the schematic image $D_{R} \subseteq \mathbb{P}_{R}^{2}$ in $\mathscr{O}_{\mathbb{P}_{R}^{2}, \xi}$ is given by the kernel of the ring homomorphism $\mathscr{O}_{\mathbb{P}_{R}^{2}, \xi} \longrightarrow \mathscr{O}_{X_{R}, \xi}$. It contains Fitt $^{0}\left(i_{R *} \mathscr{O}_{X_{R}, \xi}\right)$, which is the principal ideal $(s f-g t)$. As the map $i_{R}$ is generically an embedding, any other element in the kernel would have to be supported at $\xi$, but as the ideal $(s f-g t)$ has no embedded components the equality of ideals $I=(s f-t g)$ follows. So the matrix appearing in (3.5.3) also defines a presentation of $i_{R *} \mathscr{O}_{X_{R}, \xi}$ as $\mathscr{O}_{D_{R}, \xi}$-module and the ideal $\mathfrak{n}_{R}=(s, t)$ is the annihilator ideal $\mathfrak{n}_{R}=\operatorname{Ann}_{D_{R}}\left(i_{R *} \mathscr{O}_{X_{R}, \xi} / \mathscr{O}_{D_{R}, \xi}\right)$. Furthermore, as the elements $s$ and $t$ specialize to $x$ and $y$ we have that $\mathscr{O}_{\mathbb{P}^{2}, \xi} / \mathfrak{n}_{R}=R$. Thus $\mathfrak{n}_{R}$ is flat. By the condition (R.C.) the element $u \in i_{R *} \mathscr{O}_{X_{R}, \xi}$ corresponds to an endomorphism $\mu_{u}: \mathfrak{n}_{R} \longrightarrow \mathfrak{n}_{R}$, with $\mu_{u}(s)=-g$ and $\mu_{u}(t)=-f$. This means that $g, f \in \mathfrak{n}_{R}$.

Corollary 3.6. Let I be the defining ideal of $\operatorname{Spec}\left(\mathscr{O}_{D_{R}, \xi}\right)$ in $\operatorname{Spec}\left(\mathscr{O}_{\mathbb{R}_{R}^{2}, \xi}\right)$. Then we have that

(1) The ideal $I=\operatorname{Fitt}^{0}\left(i_{R *} \mathscr{O}_{X_{R}, \xi}\right)$. In particular, the ideal $I$ is principal and we have an inclusion of ideals $I \subseteq \mathfrak{n}_{R}^{2}$.

(2) The R-algebra $\mathscr{O}_{D_{R}, \xi}$ is flat.

(3) The scheme defined by the annihilator ideal $\operatorname{Ann}\left(i_{R *} \mathscr{O}_{X_{R}} / \mathscr{O}_{D_{R}}\right)$ in $\operatorname{Spec}\left(\mathscr{O}_{D_{R}, \xi}\right)$ is isomorphic to Spec $R$.

Proof. All three assertions are established in the proof of the above proposition.

Remark 3.7. The Ring Condition (R.C.) for the ideal $\mathfrak{n}$ or $\mathfrak{n}_{R}$ is equivalent to the condition that the entries of the first row of the matrix in (3.5.2) or (3.5.1) lie in the ideal $\mathfrak{n}$, respectively $\mathfrak{n}_{R}$. This is the content in this special case of Catanese's Rank Condition (R.C.) [Cat84]; for the terminology see also [dJvS90, Remark 1.13].

\section{Singular sections of cubics}

We give in this section an explicit description of the space of CM-curves in the plane having Hilbert polynomial $3 t+1$. The space of such CM-curves is identified with plane cubics together with a singular section.

\subsection{Critical locus}

Let $\varphi: X \longrightarrow S$ be a flat morphism of schemes, of pure relative dimension $d$. The critical locus of the morphism $\varphi$ is the closed subscheme $C(\varphi) \subseteq X$ given by the ideal sheaf Fitt ${ }^{d}\left(\Omega_{X / S}^{1}\right)$, where $\Omega_{X / S}^{1}$ is the sheaf of differentials, see [Tei77]. A section $\sigma: S \longrightarrow X$ of the morphism $\varphi: X \longrightarrow S$ is a singular section if it factorizes through the critical locus $C(\varphi)$. Thus, in the commutative diagram

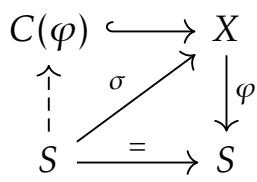

the singular section is represented by a dashed arrow. 
Example 4.2. Let $R$ be a ring, and let $f \in R\left[x_{1}, \ldots, x_{n}\right]$ be an element of the polynomial ring defining a flat family $X$ of hypersurfaces over $\operatorname{Spec}(R)=S$. One then computes, see e.g. [Tei77, Example 1, p. 588] that the $(n-1)^{\text {th }}$ Fitting ideal of $\Omega_{X / S}$ is generated by the partial derivatives of $f$. Thus we have that the ideal of the critical locus is

$$
\left(f, \frac{\partial f}{\partial x_{1}}, \ldots, \frac{\partial f}{\partial x_{n}}\right) \subseteq R\left[x_{0}, \ldots, x_{n}\right]
$$

\subsection{Singular cubics}

Let $S C$ denote the functor parametrising cubics in $\mathbb{P}^{2}$ with a singular section. That is, the $S$-valued points of $S C$ are pairs $(D, \sigma)$ where $D \subseteq \mathbb{P}_{S}^{2}=\mathbb{P}^{2} \times S$ is a flat family of cubics over $S$, and where $\sigma: S \longrightarrow D$ is a singular section.

Lemma 4.4. The critical locus $C(\varphi)$ of the universal family of cubics $\varphi: Z \subseteq \mathbb{P}^{2} \times \mathrm{Hilb}_{\mathbb{P}^{2}}^{3 t} \longrightarrow \mathrm{Hilb}_{\mathbb{P}^{2}}^{3 t}$ represents the functor $S C$. The scheme $C(\varphi)$ is smooth, projective and of dimension 8.

Proof. Let $S$ be a scheme, and let $s: S \longrightarrow C(\varphi)$ be a morphism. The morphism $s$ is determined by a pair $(\sigma, t)$, where $\sigma: S \longrightarrow \mathbb{P}^{2}$ and $t: S \longrightarrow{\text { Hilb } \mathbb{P}^{2}}_{3 t}^{3 t}$ are morphisms that together factorize through the closed subscheme $C(\varphi) \subseteq \mathbb{P}^{2} \times$ Hilb $_{\mathbb{P}^{2}}^{3 t}$. The morphism $t: S \longrightarrow$ Hilb $_{\mathbb{P}^{2}}^{3 t}$ is equivalent with having a cubic $D_{S} \subseteq \mathbb{P}_{S}^{2}=\mathbb{P}^{2} \times S$, flat over $S$. The morphism $\sigma: S \longrightarrow \mathbb{P}^{2}$ is the same as having a section of $\mathbb{P}_{S}^{2} \longrightarrow S$. Now, as our pair $(\sigma, t)$ is a point of the critical locus $C(\varphi)$ it means that the partial derivatives of the cubic vanish over $\sigma$. In other words the section $\sigma: S \longrightarrow \mathbb{P}_{S}^{2}$ factors through the critical locus of the cubic $D_{S} \longrightarrow S$. And conversely, given a flat family $D \subseteq \mathbb{P}_{S}^{2}$ of cubics over $S$ with singular section $\sigma: S \longrightarrow D \subseteq \mathbb{P}_{S}^{2}$ we obtain morphisms $\sigma: S \longrightarrow \mathbb{P}^{2}$ and $t: S \longrightarrow \operatorname{Hilb}_{\mathbb{P}^{2}}^{3 t}$ that together factorize through $C(\varphi)$.

From Example 4.2 we get that the ideal of the critical locus is locally defined by the partial derivatives of the cubic. It follows from local calculations that $C(\varphi)$ is smooth of dimension $11-3=8$.

Proposition 4.5. The functor $\mathrm{CM}_{\mathbb{P}^{2}}^{3 t+1}$ of CM-curves in $\mathbb{P}^{2}$ having Hilbert polynomial $3 t+1$ is isomorphic to the functor SC of cubics with singular section. In particular $\mathrm{CM}_{\mathbb{P}^{2}}^{3 t+1}$ is represented by the scheme $C(\varphi)$, given as the critical locus of the universal family of cubics in the plane.

Proof. First we define a morphism $\phi: \mathrm{CM}_{\mathbb{P}^{2}}^{3 t+1} \longrightarrow S C$. Given a scheme $S$ and $i_{S}: X_{S} \longrightarrow \mathbb{P}_{S}^{2}$ an $S$-valued point of $\mathrm{CM}_{\mathbb{P}^{2}}^{3 t+1}$, we let $D_{S} \subseteq \mathbb{P}_{S}^{2}$ be the schematic image of $X_{S}$ and $N_{S} \subseteq D_{S}$ the subscheme defined by the annihilator of $i_{S *} \mathscr{O}_{X_{S}} / \mathscr{O}_{D_{S}}$. Let $R$ be the local ring of a closed point of $S$ and denote by $i: X \longrightarrow \mathbb{P}^{2}$ the curve over this closed point. By Proposition 2.9 the image $D=i(X)$ is a cubic curve and there is one unique point $P \in D$ where the induced map $i: X \longrightarrow D$ is not an isomorphism. The point $P$ is a plain double point. By Corollary 3.6 we have that the schematic image $D_{R} \subseteq \mathbb{P}_{R}^{2}$ is flat over $R$, and that the subscheme $N_{R} \subseteq D_{R}$ defined by the annihilator of $i_{R *} \mathscr{O}_{X_{R}} / \mathscr{O}_{D_{R}}$, determines a singular section of $D_{R} \longrightarrow \operatorname{Spec}(R)$. Thus $\left(D_{S}, N_{S}\right)$ is a flat family of cubics in $\mathbb{P}^{2}$ with a singular section.

Next we define a morphism $\theta: S C \longrightarrow \mathrm{CM}_{\mathbb{P}^{2}}^{3 t+1}$. Let $\left(D_{S}, N_{S}\right)$ be a flat family of cubics in $\mathbb{P}^{2}$ with a singular section. We have that $D_{S}$ is given by a cubic form $Q \in \mathscr{O}_{S}[x, y, w]$. Consider an open affine set $U \subset S$ on which the section $N_{U}$ is given by two linear independent forms $s$ and $t$. As the section is singular we have that the cubic form $Q \in \mathscr{O}_{U}[x, y, w]$ can be written as $Q=s^{2} f_{1}+s t\left(f_{2}-g_{1}\right)-t^{2} g_{2}$. Consider now a matrix factorisation of $Q$

$$
M=\left(\begin{array}{cc}
g_{1} s+g_{2} t & f_{1} s+f_{2} t \\
s & t
\end{array}\right) .
$$

We can view the matrix $M$ as the presentation matrix of a sheaf $\mathscr{F}_{U}$ on $\mathbb{P}_{U}^{2}$. That is, we have the global presentation

$$
\oplus_{i=1}^{2} \mathscr{O}_{\mathbb{P}_{U}^{2}}(-2) \stackrel{M}{\longrightarrow} \mathscr{O}_{\mathbb{P}_{U}^{2}} \oplus \mathscr{O}_{\mathbb{P}_{U}^{2}}(-1) \longrightarrow \mathscr{F}_{U} \rightarrow 0 .
$$


With generators $1, u$ of $\mathscr{F}_{U}$ we have the relations $\left(u+g_{1}\right) s+g_{2} t=0$ and $f_{1} s+\left(u+f_{2}\right) t=0$. By formally eliminating $s$ and $t$ we obtain a third relation $\left(u+f_{2}\right)\left(u+g_{1}\right)=g_{2} f_{1}$. These relations can be written as the maximal minors of the matrix

$$
\left(\begin{array}{ccc}
s & -g_{2} & u+f_{2} \\
t & u+g_{1} & -f_{1}
\end{array}\right)
$$

The maximal minors define an arithmetically Cohen-Macaulay curve $X_{U} \subset \mathbb{P}_{U}^{3}=U \times \operatorname{Proj}(k[x, y, u, w])$, flat over $U$. For each point in $U$, the fiber is a curve with Hilbert polynomial $3 t+1$, and the curve does not pass through the point $(0: 0: 1: 0)$. Projection from $(0: 0: 1: 0)$ induces a map $i_{U}: X_{U} \longrightarrow \mathbb{P}_{U}^{2}$, that is an isomorphism onto its image $D_{U}$ outside the section $N_{U}$.

A different choice of the sections $s$ and $t$ and of the matrix factorization gives a curve isomorphic to $X_{U}$. The image of $X_{U}$ in $\mathbb{P}_{U}^{2}$ and the section $N_{U}$ are independent of these choices, and it follows that the curves defined for different open sets $U \subseteq S$ glue together to a curve $i_{S}: X_{S} \longrightarrow \mathbb{P}_{S}^{2}$, and thus an $S$-valued point of $\mathrm{CM}_{\mathbb{P}^{2}}^{3 t+1}$.

We next show that the two morphisms constructed are inverse of each other. For this we may assume that the base is the spectrum of a local ring $R$. Let $\left(D_{R}, N_{R}\right)$ be a flat family of cubics with a singular section. Let $i_{R}: X_{R} \longrightarrow \mathbb{P}_{R}^{2}$ be the CM-curve we get by applying the morphism $\theta$ to the pair $\left(D_{R}, N_{R}\right)$. From the construction we have that $\phi \circ \theta$ is the identity, and we verify that $\theta \circ \phi$ is the identity. Outside the section $N_{R}$ the two curves $X_{R}$ and $D_{R}$ are isomorphic, so in particular $X_{R} \backslash i_{R}^{-1}\left(N_{R}\right)$ is determined by the pair $\left(D_{R}, N_{R}\right)$. Over the closed fiber we have that the non-isomorphism locus is one point $P \in \mathbb{P}^{2}$, and we let $\xi \in \mathbb{P}_{R}^{2}$ be the image of $P$ under the natural inclusion. By Proposition 3.5 we have that the ideal $\mathfrak{n}_{R}$ of the section $N_{R} \subset D_{R}$ is contained in the ideal defining $\xi$. Let $A=\mathscr{O}_{R, \xi}$ and $B=\left(i_{R *} \mathscr{O}_{X_{R}}\right)_{\xi}$. The last statement of Proposition 3.5 tells us that the ideal $\mathfrak{n}_{R}$ is the annihilator ideal $\operatorname{Ann}_{A}(B / A)$. Hence $\mathfrak{n}_{R}$ is the dual module $\operatorname{Hom}_{A}(B, A)$. By Proposition 3.4 we then have that the $A$-algebra $B$ is the endomorphism ring $\operatorname{Hom}_{A}\left(\mathfrak{n}_{R}, \mathfrak{n}_{R}\right)$. Thus the structure sheaf $i_{R_{*}} \mathscr{O}_{X_{R}}$ is determined, up to isomorphism, by the pair $\left(D_{R}, N_{R}\right)$. It follows that $\theta \circ \phi$ is the identity.

Remark 4.6. The proof could have been shortened using Zariski Main Theorem. We have chosen to give an explicit proof that we believe is more illuminating.

Remark 4.7. There is a natural map from $C(\varphi)$ to $\operatorname{Hilb}_{\mathbb{P}^{2}}^{3 t}=\mathbb{P}^{9}$, obtained by composing the closed immersion with the projection. This is a map from the space of cubics with a singular section $S C$ to the Hilbert scheme forgetting the singular section. The image will be the set of singular cubics in the plane. Outside the locus of cubics with multiple components the map is the normalization morphism, see [Tei77, Theorem 5.5.1]. Over the set corresponding to multiple components the map is a proper modification.

\subsection{Remarks on conics}

It turns out that the situation with CM-curves in $\mathbb{P}^{2}$ having Hilbert polynomial $2 t+2$ can be treated quite analogously to the situation with twisted cubics. For readability we did not merge those similar arguments in the above text. We claim that they show that the space $\mathrm{CM}_{\mathbb{P}^{2}}^{2 t+2}$ of $\mathrm{CM}$-curves in the plane having Hilbert polynomial $2 t+2$ is isomorphic to the space of plane conics with a singular section.

\section{The space of twisted cubics}

This section contains our main contribution that identifies the space of CM-curves in $\mathbb{P}^{3}$ having Hilbert polynomial $3 t+1$ with one specific component of the Hilbert scheme of twisted cubics in $\mathbb{P}^{3}$. We also treat the case $n>3$. 
Lemma 5.1. Let $i_{R}: X_{R} \longrightarrow \mathbb{P}_{R}^{3}$ be an element of $\mathrm{CM}_{\mathbb{P}^{3}}^{3 t+1}(\operatorname{Spec}(R))$, where $R$ is a local ring. Suppose that the induced map $i: X \longrightarrow \mathbb{P}^{3}$ over the closed point in $R$ is not a closed immersion. Then the closed subscheme $Z \subseteq \mathbb{P}_{R}^{3}$ defined by the $0^{\text {th }}$ Fitting ideal sheaf of $i_{R_{*}} \mathscr{O}_{X_{R}}$ is flat over $\operatorname{Spec}(R)$, with Hilbert polynomial $3 t+1$.

Proof. The image of the CM-curve $i: X \longrightarrow \mathbb{P}^{3}$ over the closed fiber is by Proposition 2.9 a plane cubic. We may assume that the image lies in the plane $\Pi=\{z=0\} \subset \mathbb{P}^{3}=\operatorname{Proj}(k[x, y, z, w])$ and that the plain double point is $(0: 0: 0: 1)$. From Proposition 2.11 it follows that we may assume that the curve $X$ lies in $\mathbb{P}^{3}=\operatorname{Proj}(k[x, y, u, w])$ and that the morphism $i$ is induced by the linear map that sends $(x, y, z, w)$ to $(x, y, 0, w)$. In particular we may assume that the curve $X$ does not pass through the point $(0: 0: 1: 0)$. We compose the map $i_{R}: X_{R} \longrightarrow \mathbb{P}_{R}^{3}$ with the rational projection $\mathbb{P}_{R}^{3} \rightarrow \Pi_{R}$. This provides us with an element of $\mathrm{CM}_{\Pi}^{3 t+1}(R)$ and the image of $X_{R}$ is by Proposition 4.5 a cubic $D_{R} \subset \Pi_{R}$ together with a singular section $N_{R}$. After a change of coordinates we may assume that the section is given by $s=x$ and $t=y$. Then the maximal minors of (4.5.2) (with $f_{2}=0$ ) that generate the ideal of $X_{R}$ in $\mathbb{P}^{3}=\operatorname{Proj}(k[x, y, u, w]$ ) are

$$
\left(x u+g, y u+f, u\left(u+g_{1}\right)-f_{1} g_{2}\right),
$$

where $g=g_{1} x+g_{2} y$ and $f=x f_{1}$. The cubic $D_{R} \subset \Pi_{R}$ is given by $Q=f_{1} x^{2}-g_{1} x y-g_{2} y^{2} \in R[x, y, w]$. The map $i_{R}: X_{R} \longrightarrow \mathbb{P}_{R}^{3}$ is then obtained from the linear map $i^{*}$ that sends $z$ to $\alpha w+\beta u+\gamma y+\delta x$ with $\alpha, \beta$, $\gamma$ and $\delta$ in the maximal ideal of $R$. By a coordinate change in the image, making $z-\alpha w-\gamma y-\delta x$ the new $z$-coordinate we bring the map into the final form: $i^{*}$ sends $z$ to $\beta u$ and is the identity on $x, y$ and $w$.

We now compute a presentation of $X_{R}$ over $\mathbb{P}_{R}^{3}$. In (4.5.1) we have a presentation of $X_{R}$ over $\mathbb{P}_{R}^{2}$. We identify the plane with the subscheme in $\mathbb{P}_{R}^{3}$ defined by $z=0$. We have that $i_{*} \mathscr{O}_{X_{R}}$ is generated by two elements 1 and $u$ over $\mathscr{O}_{\mathbb{P}_{R}^{3}}$. To obtain a presentation we only need to add the relations coming from the action of $z$. We have that $z \cdot 1=\beta u$, and therefore $z \cdot u=\beta u^{2}$. We rewrite $\beta u^{2}$ using the relation $u^{2}+u g_{1}-f_{1} g_{2}=0$. Therefore we have the presentation

$$
\mathscr{O}_{\mathbb{P}^{3}}(-1) \oplus 3 \mathscr{O}_{\mathbb{P}^{3}}(-2) \stackrel{M}{\longrightarrow} \mathscr{O}_{\mathbb{P}^{3}} \oplus \mathscr{O}_{\mathbb{P}^{3}}(-1) \longrightarrow i_{*} \mathscr{O}_{X_{R}} \longrightarrow 0
$$

with $M$ the matrix

$$
\left(\begin{array}{cccc}
z & -\beta f_{1} g_{2} & g_{1} x+g_{2} y & f_{1} x \\
-\beta & z+\beta g_{1} & x & y
\end{array}\right)
$$

A computation shows that the $0^{\text {th }}$ Fitting ideal $I=\operatorname{Fitt}^{0}\left(i_{*} \mathscr{O}_{X_{R}}\right)$ is generated by four elements $Q, F_{1}, F_{2}$, and $F_{3}$, where $Q=Q(x, y, w)$ is our recurring cubic, and where

$$
\begin{aligned}
& F_{1}=z^{2}+\beta g_{1} z-\beta^{2} f_{1} g_{2} \\
& F_{2}=z x+\beta g_{1} x+\beta g_{2} y \\
& F_{3}=z y+\beta f_{1} x .
\end{aligned}
$$

As $R[x, y, w] /(Q)$ is a flat family of cubics the $R$-module of degree $n$ forms is locally free of rank $3 n$. From the leading terms of $F_{1}, F_{2}$ and $F_{3}$ it follows that the $R$-module of degree $n$ forms in the graded quotient ring $R[x, y, z, w] /\left(Q, F_{1}, F_{2}, F_{3}\right)$ is locally free of rank $3 n+1$, adding only the free component with basis $z w^{n-1}$ to the forms determined by the cubic $Q$ alone. Therefore $Z$ is flat over $R$.

\subsection{The twisted cubic component}

The Hilbert scheme Hilb $\mathbb{P}^{3}$ of closed subschemes in $\mathbb{P}^{3}$ having Hilbert polynomial $3 t+1$ consists of two smooth components. This was proven by Piene and Schlessinger [PS85] when the base field has characteristic different from 2 and 3, but their result is valid in any characteristic, see Proposition A.1. One of these components is 12 dimensional and contains an open subset that parametrizes twisted cubics in $\mathbb{P}^{3}$. We refer to the component $\mathrm{H}$ as the twisted cubic component. 
Theorem 5.3. Let $\mathrm{CM}_{\mathbb{P}^{3}}^{3 t+1}$ be the space of CM-curves in $\mathbb{P}^{3}$ with Hilbert polynomial $3 t+1$, and let $\mathrm{H} \subset \mathrm{Hilb}_{\mathbb{P}^{3}}^{3 t+1}$ denote the twisted cubic component of the Hilbert scheme. We have an isomorphism

$$
\phi: \mathrm{CM}_{\mathbb{P}^{3}}^{3 t+1} \longrightarrow \mathrm{H},
$$

mapping an S-valued point $(X, i)$ to the closed subscheme in $\mathbb{P}_{S}^{3}$ determined by the $0^{\text {th }}$ Fitting ideal Fitt $^{0}\left(i_{*} \mathscr{O}_{X}\right)$.

Proof. To show that the morphism $\phi$ is well-defined it suffices to check it over an affine base scheme $S=\operatorname{Spec}(R)$. If the map $i_{R}: X_{R} \longrightarrow \mathbb{P}_{R}^{3}$ is a closed immersion there is nothing to prove as the $0^{\text {th }}$ Fitting ideal is the the defining ideal of the image of the closed immersion. If the map is not a closed immersion it follows from Lemma 5.1 that the $0^{\text {th }}$ Fitting ideal defines a flat subscheme with appropriate Hilbert polynomial. We have therefore a morphism $\phi$ from $\mathrm{CM}_{\mathbb{P}^{3}}^{3 t+1}$ to the Hilbert scheme $\mathrm{Hilb}_{\mathbb{P}^{3}}^{3 t+1}$. As $\mathrm{CM}_{\mathbb{P}^{3}}^{3 t+1}$ is irreducible it follows that the morphism factorizes through the twisted cubic component $\mathrm{H}$ and gives the sought morphism.

Let $Z \subseteq \mathbb{P}^{3}$ be a closed subscheme corresponding to a point on the twisted cubic component $\mathrm{H}$. There is a divisor $\delta$ on $\mathrm{H}$ corresponding to singular planar cubics together with a spatial embedded point. If $Z$ corresponds to a point on $\mathrm{H}$ but not on the divisor $\delta$ then the closed immersion $i: Z \longrightarrow \mathbb{P}^{3}$ is a CM-curve. If $Z$ is a point on the divisor, then it is a plane cubic, lying in a plane $\Pi \subset \mathbb{P}^{3}$, with an embedded point at a singular point $P$ of the cubic, see ([PS85, Lemma 2]). By Proposition 4.5 there is a (unique) CM-curve $i_{\Pi}: X \longrightarrow \Pi$ such that the image of $X$ is the singular cubic and the map $i_{\Pi}$ is not an isomorphism onto its image at $P$. Let $i$ be the composed map $i: X \longrightarrow \Pi \subset \mathbb{P}^{3}$. Then the image by $\phi$ of the curve $(X, i)$ is $Z \subset \mathbb{P}^{3}$. More explicitly, the scheme $Z$ is projectively equivalent to the scheme given by the ideal $I=\left(x z, y z, z^{2}, Q\right)$ where $Q=Q(x, y, w)$ is a cubic form singular at $(0: 0: 1)$ (see [PS85, Lemma 2]). As in the proof of Lemma 5.1 the curve $X$ is given by the maximal minors of the matrix (4.5.2) and we have a presentation (5.1.1) with $\beta=0$. The $0^{\text {th }}$ Fitting ideal is the ideal $\left(Q, z^{2}, z x, z y\right)$. It follows that the morphism $\phi: \mathrm{CM} \longrightarrow \mathrm{H}$ is bijective. Because the spaces are isomorphic on an open dense subset, the isomorphism follows from Zariski's Main Theorem [Mum88, Chapter III, §9] as both spaces are smooth of dimension 12.

Remark 5.4. Freiermuth and Trautmann studied the moduli scheme of stable sheaves supported on cubic space curves [FT04]. In characteristic zero there exists a projective coarse moduli space $M_{X}^{p}$ for semi-stable sheaves on a smooth projective variety $X$ with a fixed Hilbert polynomial $p$. For $p(t)=3 t+1$ and $X=\mathbb{P}^{3}$ all sheaves in $M=M_{\mathbb{P}^{3}}^{3 t+1}$ are stable. The result of [FT04] is that the projective variety $M$ consists of two nonsingular, irreducible, rational components $M_{0}$ and $M_{1}$ of dimension 12 and 13, intersecting transversally in a smooth variety of dimension 11, see also Appendix B.

The component $M_{0}$ is isomorphic to the twisted cubic component $\mathrm{H}$ of the Hilbert scheme. The identification also uses Fitting ideals. If $i: X \longrightarrow \mathbb{P}^{3}$ is a CM-curve, with Hilbert polynomial $3 t+1$, then the module $i_{*} \mathscr{O}_{X}$ is a stable sheaf supported on a cubic. Thus, by forgetting the algebra structure of $i_{*} \mathscr{O}_{X}$ we get that our morphism $\phi: \mathrm{CM}_{\mathbb{P}^{3}}^{3 t+1} \longrightarrow \mathrm{H}$ factorizes through the moduli scheme of stable sheaves.

Example 5.5. The following example shows that the $0^{\text {th }}$ Fitting ideal does not always give a morphism from $\mathrm{CM}$ to the Hilbert scheme. Indeed, we show that here the family of closed subschemes determined by the $0^{\text {th }}$ Fitting ideal is not flat.

We start with a genus 2 curve embedded with the linear system $|5 P|$, where $P$ is a Weierstrass point. More precisely, we look at the curve $X \subset \mathbb{P}^{3}$ given by the homogeneous ideal generated by the maximal minors of

$$
\left(\begin{array}{ccc}
x & u & y^{2}+w^{2} \\
y & x & u^{2}
\end{array}\right)
$$

The projection from $X$ to the plane $\mathbb{P}^{2}$ in the coordinates $x, y, w$ is finite. Let another $\mathbb{P}^{3}$ be given by the homogenous coordinate ring $k[x, y, z, w]$, and consider the family of maps $i_{t}: X_{t} \cong X \longrightarrow \mathbb{P}^{3}, t \in T=\mathbb{A}^{1}$, determined by the linear map sending $(x, y, z, w)$ to $(x, y, t u, w)$. 
In the affine chart $\{w=1\}$ we get that $i_{t *} \mathscr{O}_{X}$ has presentation

$$
\left(\begin{array}{cccccc}
z & 0 & -t x\left(y^{2}+1\right) & 0 & x^{2} & -y-y^{3} \\
-t & z & 0 & x^{2} & -y & 0 \\
0 & -t & z & -y & 0 & x
\end{array}\right)
$$

The $0^{\text {th }}$ Fitting ideal is then the ideal generated by

$$
\begin{array}{cc}
z^{3}-t^{3} x\left(y^{2}+1\right), & z^{2} x-t^{2} y\left(y^{2}+1\right), \\
z x^{3}-t y^{2}\left(y^{2}+1\right), & x^{5}-y^{3}\left(y^{2}+1\right), \\
\left(y z-t x^{2}\right) x, \quad\left(y z-t x^{2}\right) y, & \left(y z-t x^{2}\right) z, \quad\left(y z-t x^{2}\right) t .
\end{array}
$$

The family determined by this ideal is not flat, as $y z-t x^{2}$ is a $t$-torsion element. For $t \neq 0$ we have the generator $y z-t x^{2}$ in the ideal, making the three first generators on the last row above superfluous.

The family above gives an $T=\mathbb{A}^{1}$-valued point $\left(X_{T}=X \times \mathbb{A}^{1}, i_{T}\right)$ of $\mathrm{CM}_{\mathbb{P}^{3}}^{5 t-1}$. Then by taking the $0^{\text {th }}$ Fitting ideal of $i_{T *} \mathscr{O}_{X_{T}}$ we get a closed subscheme $Z \subseteq \mathbb{P}_{\mathbb{A}^{1}}^{3}$ which is not a flat family, and in particular the Hilbert polynomial of a fiber is not constant.

\subsection{Higher codimension}

The twisted cubic component $\mathrm{H}_{n}$ of the Hilbert scheme Hilb $\mathrm{P}^{n}{ }^{3 t+1}$ for $n>3$ has been described by Chung and Kiem [CK11]; their proof works in any characteristic. The component $\mathrm{H}_{n}$ is isomorphic to a component of the relative Hilbert scheme of the $\mathbb{P}^{3}$-bundle $\mathbb{P} \mathscr{U} \longrightarrow G_{4}^{n+1}$, where $\mathscr{U}$ is the universal rank 4 vector bundle on the Grassmannian $G_{4}^{n+1}$ [CK11, Proposition 3.3]. Chung and Kiem also describe a morphism $\mathrm{H}_{n} \longrightarrow M_{0}$ to a component of the moduli scheme $M_{\mathbb{P}^{n}}^{3 t+1}$ of stable sheaves and show that this morphism realises $\mathrm{H}_{n}$ as the blow-up of $M_{0}$ along the smooth locus of stable sheaves with planar support [CK11, Proposition 1.3]. We have a similar result for the space of CM-curves.

We remark that the construction with the Fitting ideal does not give a morphism from $\mathrm{CM}_{\mathbb{P}^{n}}^{3 t+1}$ to Hilb $_{\mathbb{P}^{n}}^{3 t+1}$ if $n>3$. Indeed, if the image of a CM-curve $X \longrightarrow \mathbb{P}^{n}$ is a planar curve then the $0^{\text {th }}$ Fitting ideal gives a scheme with Hilbert polynomial $3 t+n-2$. By a coordinate transformation we may assume that the plane containing the image $i(X)$ is given by $z_{1}=\cdots=z_{n-2}=0$ and that the non-isomorphism locus on the

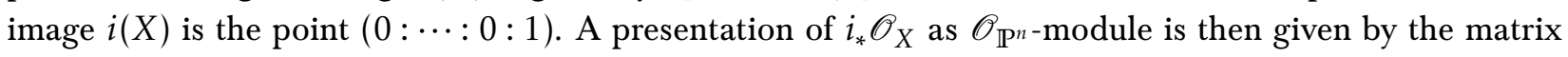

$$
\left(\begin{array}{ccccccc}
z_{1} & 0 & \ldots & z_{n-2} & 0 & g & f \\
0 & z_{1} & \ldots & 0 & z_{n-2} & x & y
\end{array}\right)
$$

The $0^{\text {th }}$ Fitting ideal is the ideal $I=\left(z_{i}^{2}, z_{i} z_{j}, z_{i} x, z_{i} y, y g-x f\right)$ for $1 \leq i \leq n-2$ and $i<j \leq n-2$. There is a $\mathbb{P}^{n-3}$ of $\mathbb{P}^{3}$ 's containing the plane $z_{1}=\cdots=z_{n-2}=0$ and each of these $\mathbb{P}^{3}$ 's contains a subscheme $Z$ with Hilbert polynomial $3 t+1$ with the planar singular cubic as 1-dimensional subscheme.

Proposition 5.7. The twisted cubic component $\mathrm{H}_{n}$ of the Hilbert scheme $\mathrm{Hilb}_{\mathbb{P}^{n}}^{3 t+1}, n>3$, is isomorphic to the blow-up of $\mathrm{CM}_{\mathbb{P}^{n}}^{3 t+1}$ in the locus of CM-curves with planar scheme-theoretic image.

Proof. Let $\left(X_{S}, i_{S}\right)$ be an $S$-valued point of $\mathrm{CM}=\mathrm{CM}_{\mathbb{P}^{n}}^{3 t+1}$. By Proposition 2.11 we have that $H^{0}\left(X_{S}, i_{S}^{*} \mathscr{O}_{\mathbb{P}_{S}^{n}}(1)\right)$ is a locally free $\mathscr{O}_{S}$-module of rank 4 . Let $G=G_{4}^{n+1}$ be the Grassmannian of rank 4 quotients of the free module $H^{0}\left(\mathbb{P}^{n}, \mathscr{O}(1)\right)$ of rank $n+1$. We define the subfunctor $\widetilde{\mathrm{CM}}$ of $\mathrm{CM} \times G$ by setting, for any scheme $S$

$$
\widetilde{\mathrm{CM}}(S)=\left\{\left(\left(X_{S}, i_{S}\right), Q_{S}\right) \mid i_{S}^{*} \text { factors through } Q_{S}\right\} \text {. }
$$

The condition that $i_{S}^{*}: H^{0}\left(\mathbb{P}_{S}^{n}, \mathscr{O}_{\mathbb{P}_{S}^{n}}(1)\right) \longrightarrow H^{0}\left(X_{S}, i_{S}^{*} \mathscr{O}_{\mathbb{P}_{S}^{n}}(1)\right)$ factors through $H^{0}\left(\mathbb{P}_{S}^{n}, \mathscr{O}_{\mathbb{P}_{S}^{n}}(1)\right) \longrightarrow Q_{S}$ is a closed condition on $S$. And consequently $\widetilde{\mathrm{CM}}$ is represented by a closed subspace of the product. We have that the projection $\sigma: \widetilde{\mathrm{CM}} \longrightarrow \mathrm{CM}$ is an isomorphism over the open set where the corresponding curves $i_{S}: X_{S} \longrightarrow \mathbb{P}_{S}^{n}$ are closed immersions. 
To study this morphism $\sigma$ we first describe $\mathrm{CM}$ in a neighbourhood of a curve $i: X \longrightarrow \mathbb{P}^{n}$ whose image is a planar curve. We may assume that the plane is given as $z_{1}=\cdots=z_{n-2}=0$, that $X$ is given by an ideal $I$ in $k[x, y, u, w]$, and that the map $i$ is given by $i^{*}\left(x, y, z_{1}, \ldots, z_{n-2}, w\right)=(x, y, 0, \ldots, 0, w)$. It follows from Lemma 2.15 and by arguing as in the proof of Lemma 5.1 that we may assume that a neighborhood of the curve $i: X \longrightarrow \mathbb{P}^{n}$ is given by perturbing the ideal $I \subset k[x, y, u, w]$ and perturbing the map $i^{*}$ to the map $j^{*}$ that sends $\left(x, y, z_{1}, \ldots, z_{n-2}, w\right)$ to $\left(x, y, b_{1,1} x+b_{1,2} y+b_{1,3} w+b_{1,4} u, \ldots, b_{n-2,1} x+b_{n-2,2} y+b_{n-2,3} w+b_{n-2,4} u, w\right)$. The locus of CM-curves with planar scheme-theoretic image is locally given by the equations

$$
b_{1,4}=b_{2,4}=\cdots=b_{n-2,4}=0 .
$$

An affine chart of the Grassmannian is obtained by choosing four global sections of $H^{0}\left(\mathbb{P}^{n}, \mathscr{O}_{\mathbb{P}^{n}}(1)\right)$ that form a basis of $H^{0}\left(X, i^{*} \mathscr{O}_{\mathbb{P}^{n}}(1)\right)$. The affine chart is then the affine space representing linear maps from the remaining $n-3$ global sections to $H^{0}\left(X, i^{*} \mathscr{O}_{\mathbb{P}^{n}}(1)\right)$. By choosing the global sections $x, y, w, z_{1}$ the universal family over the affine chart is then the map that sends $z_{i} \mapsto \lambda_{i, 1} x+\lambda_{i, 2} y+\lambda_{i, 3} w+\lambda_{i, 4} z_{1}$, for every $i=2, \ldots, n-2$. The condition that the map $j^{*}$ factors through the quotient map on the affine chart of the Grassmannian is that $b_{k, m}=\lambda_{k, m}+\lambda_{k, 4} b_{1, m}$ for $2 \leq k \leq n-2, m=1,2,3$ and $b_{k, 4}=\lambda_{k, 4} b_{1,4}$ for $2 \leq k \leq n-2$. The last $n-3$ equations show that the morphism $\sigma$ is the blow up of the locus of CM-curves with planar scheme-theoretic image.

Let $\mathscr{U}$ be the universal quotient bundle over $G$. By [CK11] the Hilbert scheme $\mathrm{Hilb}_{\mathbb{P}^{n}}^{3 t+1}$ is isomorphic to the relative Hilbert scheme Hilb $\operatorname{PP\mathscr {U}}^{3 t+1} \longrightarrow G$, with fibres isomorphic to $\mathrm{Hilb}_{\mathbb{P}^{3}}^{3 t+1}$. We define a morphism from $\widetilde{\mathrm{CM}}$ to the Hilbert scheme Hilb $\mathrm{P} \mathscr{U} / G^{3 t+1} \longrightarrow G$ by sending a pair $\left(\left(X_{S}, i_{S}\right), Q_{S}\right)$ to the closed subscheme in $\mathbb{P} \mathscr{U}$ determined by the $0^{\text {th }}$ Fitting ideal of $i_{S *} \mathscr{O}_{X_{S}}$ over $Q_{S}$.

By Theorem 5.3 the space of CM curves in each fibre is isomorphic to the twisted cubic component of the fibre of the Hilbert scheme $\mathrm{Hilb}_{\mathbb{P} \mathscr{U} / G}^{3 t+1} \longrightarrow G$. By [CK11] the twisted cubic component $\mathrm{H}_{n}$ is the irreducible smooth scheme in $\mathrm{Hilb}_{\mathbb{P} \mathscr{U}}^{3 t+1} \longrightarrow G$ where the fibers are twisted cubic components $\mathrm{H} \subset \mathrm{Hilb}_{\mathbb{P}^{3}}^{3 t+1}$.

\subsection{The Hilbert scheme component with two skew lines}

The Hilbert scheme $\mathrm{Hilb}_{\mathbb{P}^{3}}^{2 t+2}$ of closed subschemes in $\mathbb{P}^{3}$ over a field of characteristic zero, having Hilbert polynomial $2 t+2$ consists of two smooth components. The smoothness of these components was observed in [Har82], and a proof was given by Chen, Coskun, and Nollet in [CCN11]. One of these components $\mathrm{H}_{3}$ is smooth of dimension 8 and a general point correspond to a pair of skew lines. We claim that with the arguments presented in the present article, one can prove that we have a morphism $\phi: \mathrm{CM}_{\mathbb{P}^{3}}^{2 t+2} \longrightarrow H_{3}$ sending a $S$-valued point $(X, i)$ to the closed subscheme in $\mathbb{P}^{3} \times S$ defined by the $0^{\text {th }}$ Fitting ideal sheaf $\operatorname{Fitt}^{0}\left(i_{*} \mathscr{O}_{X}\right)$. The morphism is an isomorphism.

\section{A. The Hilbert scheme of twisted cubics}

The main purpose of this section is to prove the following result.

Proposition A.1 (Piene-Schlessinger). The Hilbert scheme $\mathrm{Hilb}_{\mathbb{P}^{3}}^{3 t+1}$ is the union of two nonsingular rational varieties $H$ and $H^{\prime}$, of dimension 12 and 15; their intersection is non-singular, transversal, and rational of dimension 11.

Remark A.2. The statement about the structure of the Hilbert scheme for algebraically closed fields of characteristic different from 2 and 3 is found [PS85]. The reason for avoiding these characteristics lies in the deformation computation in Section 5 of their paper; this is not needed for the results in the rest of the paper. Our characteristic free Lemma A.8 given below, replaces Lemma 6 in loc. cit., from where the proposition then follows. 
Remark A.3. Piene and Schlessinger reduce the computation of the local structure of the Hilbert scheme to a deformation computation for a saturated homogeneous ideal in $k[x, y, z, w]$ by their Comparison Theorem in [PS85, Section 3].

A detailed explanation of this type of deformation computation is given in [Ste95], which furthermore contains an easier example of two intersecting lines with an embedded point at the origin, relevant for the $(2 t+2)$-case.

\section{A.4. Tangent space calculations}

We start by reproving the following, known, explicit description of the following open affine chart of the Hilbert scheme Hilb ${ }^{3 t+1}$ of closed subschemes in $\mathbb{P}^{3}$ with Hilbert polynomial $3 t+1$, a result we relied on in Section 2.

Lemma A.5. Let $Z \subseteq \mathbb{P}^{3}$ be the closed subscheme given by the graded ideal $I=\left(u^{2}, u y-x^{2}, x u\right) \subseteq k[x, y, u, w]$. An affine open chart around the corresponding point in the Hilbert scheme $\mathrm{Hilb}^{3 t+1}$ is given by the polynomial ring $A=k\left[a_{1}, \ldots, a_{12}\right]$. The maximal minors of

$$
\left(\begin{array}{ccc}
x+a_{2} w & a_{7} y+a_{6} w & u+a_{12} x+a_{11} y+a_{10} u+a_{9} w \\
y+a_{1} w & u+a_{5} x+a_{4} y+a_{3} w & x+a_{8} w
\end{array}\right)
$$

generate an ideal $I(a) \subset A[x, y, u, w]$ that determines the restriction of the universal family to $\operatorname{Spec}(A)$.

Proof. The first order deformations are determined by the global sections of the normal sheaf $H^{0}\left(Z, N_{Z / \mathbb{P}^{3}}\right)$. It is convenient to work in the affine chart $\{w=1\}$. As the ideal is determinantal, we can compute as described in [Sch73]. The sections of the normal sheaf are then generated by the following six deformations induced by perturbing the matrix

$$
\left(\begin{array}{ccc}
x+\varepsilon_{11} & \varepsilon_{12} & u+\varepsilon_{13} \\
y+\varepsilon_{21} & u+\varepsilon_{22} & x+\varepsilon_{23}
\end{array}\right)
$$

We describe an infinitesimal deformation by its action on the vector $\left(u^{2}, y u-x^{2}, x u\right)$ of generators of the ideal. Written out this action reads

$$
\begin{array}{llrl}
\frac{\partial}{\partial \varepsilon_{11}}=(0,-x, u) & \frac{\partial}{\partial \varepsilon_{12}}=(-x, 0,-y) & \frac{\partial}{\partial \varepsilon_{13}}=(u, y, 0) \\
\frac{\partial}{\partial \varepsilon_{21}}=(0, u, 0) & \frac{\partial}{\partial \varepsilon_{22}}=(u, 0, x) & \frac{\partial}{\partial \varepsilon_{23}}=(0,-x, 0) .
\end{array}
$$

We can also multiply the generators by linear functions. We have to consider the action on the generators modulo the ideal $\left(u^{2}, y u-x^{2}, x u\right)$. We find that $u \frac{\partial}{\partial \varepsilon_{11}}=u \frac{\partial}{\partial \varepsilon_{21}}=u \frac{\partial}{\partial \varepsilon_{22}}=u \frac{\partial}{\partial \varepsilon_{23}}=x \frac{\partial}{\partial \varepsilon_{21}}=(0,0,0)$. The remaining actions are as follows

$$
\begin{gathered}
u \frac{\partial}{\partial \varepsilon_{13}}=-x \frac{\partial}{\partial \varepsilon_{11}}=-x \frac{\partial}{\partial \varepsilon_{23}}=y \frac{\partial}{\partial \varepsilon_{21}}=\left(0, x^{2}, 0\right) \\
x \frac{\partial}{\partial \varepsilon_{22}}=-u \frac{\partial}{\partial \varepsilon_{12}}=\left(0,0, x^{2}\right) \quad y \frac{\partial}{\partial \varepsilon_{22}}=-x \frac{\partial}{\partial \varepsilon_{12}}=\left(x^{2}, 0, y^{2}\right) \\
x \frac{\partial}{\partial \varepsilon_{13}}=-y \frac{\partial}{\partial \varepsilon_{23}}=(0, x y, 0) \quad-y \frac{\partial}{\partial \varepsilon_{12}}=\left(x y, 0, y^{2}\right)
\end{gathered}
$$

and lastly we have that $y \frac{\partial}{\partial \varepsilon_{11}}=-x \frac{\partial}{\partial \varepsilon_{13}}+x \frac{\partial}{\partial \varepsilon_{22}}$. We therefore get 6 additional first order deformations. As deformations of determinantal varieties are unobstructed we homogenize with respect to the variable $w$ and write a 12-dimensional family by choosing appropriate representatives. With new names for the deformation variables this can be presented as the claimed maximal minors.

Proposition A.6. Let $Z \subseteq \mathbb{P}^{3}$ be the closed subscheme given by the graded ideal $I=\left(z^{2}, z x, z y, Q\right) \subseteq k[x, y, z, w]$, where $Q=Q(x, y, w)$ is a cubic form. If $Q$ is singular at $(0: 0: 1)$ then the tangent space to the Hilbert scheme at $Z$ has dimension 16. If $Q$ is smooth at $(0: 0: 1)$, then the tangent space has dimension 15. 
Proof. Write $Q=Q(x, y, w)=x f-y g$ in $A=k[x, y, z, w]$. We then have the free resolution

$$
0 \longleftarrow A / I \longleftarrow A \stackrel{\varphi}{\longleftarrow} A(-2)^{3} \oplus A(-3) \stackrel{R}{\longleftarrow} A(-3)^{3} \oplus A(-4)
$$

where $\varphi=\left(z x, z y, z^{2}, Q\right)$ is the map given by the generators of the ideal. The relation matrix $R$ is then

$$
\left(\begin{array}{cccc}
0 & z & -y & -f \\
-z & 0 & x & g \\
y & -x & 0 & 0 \\
0 & 0 & 0 & z
\end{array}\right)
$$

Again it is convenient to work in the affine chart $\{w=1\}$. We compute deformations as described in [Art76, Section I.6] and [Ste95]. We obtain generators for the global sections of the normal sheaf $N_{Z / \mathbb{A}^{3}}$ as the syzygies of the transpose of the relation matrix $R$, but computed modulo the ideal $I=\left(z x, z y, z^{2}, Q\right)$. We give the generators of the normal sheaf by their action on the vector $\varphi$ of generators of the ideal. If $Q=x f-y g$ is singular at $(0: 0: 1)$ then neither $f$ nor $g$ have non-zero constant term. It follows that generators of the normal sheaf are

$$
\begin{array}{cccc}
(z, 0,0,0), & (0, z, 0,0), & (0,0, z, 0), & (0,0,0, z), \\
(0,0,0, x), & (0,0,0, y), & (x, y, 0,0), & (g, f, 0,0) .
\end{array}
$$

As the degree of a perturbation can be at most that of the element of $\varphi$, so 2, 2, 2,3 respectively, and one has to compute modulo the ideal $I$, we get 8 additional deformations by multiplying the last four generators above with the variables $x$ and $y$. It follows that the dimension of the Zariski tangent space to Hilb ${ }^{3 t+1}$ is 16. If $Q$ has linear terms, the last generator $(g, f, 0,0)$ is not present, and $(z, 0,0,0)$ is to be replaced by $(z, 0,0, f)$, and $(0, z, 0,0)$ by $(0, z, 0,-g)$. Then the dimension of the Zariski tangent space is 15 .

Remark A.7. The ideal $\left(z x, z y, z^{2}, Q\right)$ with $Q$ smooth at the origin gives a plane cubic through the origin with an embedded point at the origin, so on the curve. It is also possible to have a plane cubic and a point in its plane but not on the cubic. Such a curve is not a small deformation of a curve in the intersection of the two components of the Hilbert scheme. Consider the plane cubic $z=x^{3}+y^{3}+w^{3}=0$ and a point at $(0: 0: t: 1)$. For $t \neq 0$ the homogeneous ideal is generated by

$$
z x, z y, z(z-t w), z w^{2}-t\left(x^{3}+y^{3}+w^{3}\right)
$$

but if we specialize to $t=0$ we also need the equations $x\left(x^{3}+y^{3}+w^{3}\right)$ and $y\left(x^{3}+y^{3}+w^{3}\right)$. The result is the non-saturated ideal

$$
z x, z y, z^{2}, z w^{2}, x\left(x^{3}+y^{3}+w^{3}\right), y\left(x^{3}+y^{3}+w^{3}\right) .
$$

This is a case where the Comparison Theorem of [PS85] does not apply.

Lemma A.8. The graded ideal $I=\left(z^{2}, z x, z y, x^{3}\right) \subseteq k[x, y, z, w]$ has a universal deformation space given by the ideal

$$
J=\left(b_{12} c_{13}, b_{12} c_{14}, b_{12} c_{15}, b_{12} c_{16}\right)
$$

in $A=k\left[a_{1}, \ldots, a_{11}, b_{12}, c_{13}, \ldots, c_{16}\right]$. The universal family is given by the ideal in $(A / J)[x, y, z, w]$ generated by the four elements

$$
\begin{gathered}
\tilde{z} \tilde{x}+b_{12} \tilde{a}_{3} \tilde{x}-b_{12} \tilde{a}_{6} \tilde{y}, \quad \tilde{z} \tilde{y}-b_{12} \tilde{x}\left(\tilde{x}+a_{8} w\right), \\
\tilde{z}^{2}+c_{16} \tilde{z} w+b_{12} \tilde{a}_{3} \tilde{z}-b_{12}^{2} \tilde{a}_{6}\left(\tilde{x}+a_{8} w\right) \\
\tilde{x}^{3}+\tilde{a}_{3} \tilde{x} \tilde{y}-\tilde{a}_{6} \tilde{y}^{2}+a_{8} \tilde{x}^{2} w+c_{13} \tilde{x} w^{2}+c_{14} \tilde{y} w^{2}+c_{15} \tilde{z} w^{2}+c_{15} c_{16} w^{3},
\end{gathered}
$$

where $\tilde{x}=x+a_{2} w, \tilde{y}=y+a_{1} w, \tilde{z}=z+a_{11} w+a_{9} x+a_{10} y, \tilde{a}_{3}=a_{3} w+a_{4} y+a_{5} x$ and $\tilde{a}_{6}=a_{6} w+a_{7} y$. 
Proof. We compute again in the chart $\{w=1\}$. By Proposition A.6 there are 16 first order deformations, which we have to lift to higher order, as described in [Art76, Section I.6] and [Ste95]. We present these pertubations in the following way:

$$
\begin{gathered}
z x+a_{2} z+a_{9} x^{2}+a_{10} x y+a_{11} x \\
z y+a_{1} z+a_{9} x y+a_{10} y^{2}+a_{11} y-b_{12} x^{2} \\
z^{2}+\left(c_{16}+2 a_{11}\right) z \\
x^{3}+\left(a_{3}+a_{4} x y+a_{5} x\right) x y-\left(a_{6}+a_{7} y\right) y^{2}+\left(a_{8}+3 a_{2}\right) x^{2}+c_{13} x+c_{14} y+c_{15} z
\end{gathered}
$$

To avoid long formulas we continue the computation with less variables. First of all, we use coordinate transformations to simplify. Explicitly, we set $\tilde{x}=x+a_{2}, \tilde{y}=y+a_{1}$, and $\tilde{z}=z+a_{11}+a_{9} x+a_{10} y$. This is no loss of generality as one can substitute in the final formulas. Moreover, to ensure a finite computation, we use only deformations of negative weight. The computation in [PS85] shows that not all deformation variables occur in the equations for the base space. We put $\tilde{a}_{3}=a_{3}+a_{4} y+a_{5} x, \tilde{a}_{6}=a_{6}+a_{7} y$ and compute with the variables $\tilde{a}_{3}$ and $\tilde{a}_{6}$, We give the variable $\tilde{z}$ weight 2 enabling us to give $b_{12}$ weight 1 . The infinitesimal deformation now becomes

$$
\begin{gathered}
\tilde{z} \tilde{x}, \quad \tilde{z} \tilde{y}-b_{12} \tilde{x}^{2}, \quad \tilde{z}^{2}+c_{16} \tilde{z}, \\
\tilde{x}^{3}+\tilde{a}_{3} \tilde{x} \tilde{y}-\tilde{a}_{6} \tilde{y}^{2}+a_{8} \tilde{x}^{2}+c_{13} \tilde{x}+c_{14} \tilde{y}+c_{15} \tilde{z}
\end{gathered}
$$

We have to extend these generators of the ideal to higher order (in the deformation variables). To ensure flatness we have to lift the equations $\varphi R=0$ to $\widetilde{\varphi} \widetilde{R}=0$. We therefore compute the new relation matrix and then adjust the generators. The result of our computation is a vector $\widetilde{\varphi}$ with components

$$
\begin{gathered}
\tilde{z} \tilde{x}+b_{12} \tilde{a}_{3} \tilde{x}-b_{12} \tilde{a}_{6} \tilde{y} \\
\tilde{z} \tilde{y}-b_{12} \tilde{x}\left(\tilde{x}+a_{8}\right) \\
\tilde{z}^{2}+c_{16} \tilde{z}+b_{12} \tilde{a}_{3} \tilde{z}-b_{12}^{2} \tilde{a}_{6}\left(\tilde{x}+a_{8}\right) \\
\tilde{x}^{3}+\tilde{a}_{3} \tilde{x} \tilde{y}-\tilde{a}_{6} \tilde{y}^{2}+a_{8} \tilde{x}^{2}+c_{13} \tilde{x}+c_{14} \tilde{y}+c_{15} \tilde{z}+c_{15} c_{16}
\end{gathered}
$$

with relation matrix $\widetilde{R}$

$$
\left(\begin{array}{cccc}
-b_{12}\left(\tilde{x}+a_{8}\right) & \tilde{z}+c_{16} & -\tilde{y} & -\tilde{x}\left(\tilde{x}+a_{8}\right)-c_{13} \\
-\tilde{z}-c_{16}-b_{12} \tilde{a}_{3} & b_{12} \tilde{a}_{6} & \tilde{x} & -c_{14}-\tilde{a}_{3} \tilde{x}+\tilde{a}_{6} \tilde{y} \\
\tilde{y} & -\tilde{x} & 0 & -c_{15} \\
0 & 0 & b_{12} & \tilde{z}
\end{array}\right) .
$$

Modulo terms of degree three in the deformation variables we have

$$
\widetilde{\varphi} \widetilde{R} \equiv\left(b_{12} c_{16} \tilde{x}^{2}, 0, b_{12} c_{13} \tilde{x}+b_{12} c_{14} \tilde{y}+b_{12} c_{15} \tilde{z}, b_{12} c_{14} \tilde{x}^{2}\right) .
$$

These terms cannot be cancelled by additional higher order terms of $\varphi$, so the (quadratic) obstruction to lift the deformation is given by the ideal

$J=\left(b_{12} c_{13}, b_{12} c_{14}, b_{12} c_{15}, b_{12} c_{16}\right)$. As the product $\widetilde{\varphi} \widetilde{R}$ is in fact equal to zero modulo the ideal $J$, the family given is indeed the universal family.

Remark A.9. Compared with [PS85] our formulas differ (apart from the names and signs of the variables) in two respects. Our $a_{5}$ is not present in [PS85], as there it was transformed away by a coordinate transformation, which does not work in characteristic 3. But the main difference is the absence of a factor 2 in front of $\tilde{a}_{3}-$ it is put there in [PS85] to complete the square in the third generator, transforming the term $b_{12} \tilde{a}_{3} z$ away; this cannot be done in characteristic two.

Remark A.10. The naming of the deformation variables is chosen such that the variables $a_{1}, \ldots, a_{11}$ represent deformations in the intersection of the two components of the Hilbert scheme, the variable $b_{12}$ is in 
the direction of the twisted cubic component and the variables $c_{13}, \ldots, c_{16}$ represent deformations in the direction of the other component.

\section{B. The moduli space of stable sheaves}

In this section we relate the moduli space of stable sheaves on $\mathbb{P}^{3}$ with Hilbert polynomial $3 t+1$ to the Hilbert scheme. The definition of (semi-)stability is recalled in [FT04, Section 2]. Note that they work over a fixed, algebraically closed field of characteristic zero.

Every stable sheaf $\mathscr{F}$ on $\mathbb{P}^{3}$ with Hilbert polynomial $3 t+1$ has a free resolution of the form [FT04]

$$
0 \leftarrow \mathscr{F} \leftarrow \mathscr{O}_{\mathbb{P}^{3}} \oplus \mathscr{O}_{\mathbb{P}^{3}}(-1) \stackrel{A}{\longleftarrow} \mathscr{O}_{\mathbb{P}^{3}}(-1) \oplus 3 \mathscr{O}_{\mathbb{P}^{3}}(-2) \stackrel{B}{\longleftarrow} 2 \mathscr{O}_{\mathbb{P}^{3}}(-3) \leftarrow 0
$$

Any flat deformation of $\mathscr{F}$ is obtained by perturbing the matrices $A$ and $B$ to $\widetilde{A}$ and $\widetilde{B}$ such that $\widetilde{A B}=0$.

We consider again the most singular point on the intersection of both components of $M_{\mathbb{P}^{3}}^{3 t+1}$, which is the sheaf $\mathscr{F}=i_{*} \mathscr{O}_{X}$ with $(X, i)$ the CM-curve of Lemma 2.13. It has a presentation with matrices

$$
A=\left(\begin{array}{cccc}
z & 0 & 0 & -x^{2} \\
0 & z & x & y
\end{array}\right) \quad \text { and } \quad B=\left(\begin{array}{cc}
0 & x^{2} \\
-x & -y \\
z & 0 \\
0 & z
\end{array}\right) .
$$

Lemma B.1. Let $\mathscr{F}$ be the stable sheaf with presentation matrix A above. An affine open chart around the corresponding point in the moduli space $M_{\mathbb{P}^{3}}^{3 t+1}$, is given by the ideal $J=\left(b_{12} c_{13}, b_{12} c_{14}\right)$ in $k\left[a_{1}, \ldots, a_{11}, b_{12}, c_{13}, c_{14}\right]$. The universal family over that affine chart has a presentation with matrices

$$
\widetilde{A}=\left(\begin{array}{cccc}
\tilde{z} & -b_{12} \tilde{a}_{6}\left(\tilde{x}+a_{8}\right) & \tilde{a}_{3} \tilde{x}-\tilde{a}_{6} \tilde{y}+c_{14} & -\tilde{x}\left(\tilde{x}+a_{8}\right)-c_{13} \\
-b_{12} & \tilde{z}+b_{12} \tilde{a}_{3} & \tilde{x} & \tilde{y}
\end{array}\right)
$$

and

$$
\widetilde{B}=\left(\begin{array}{cc}
-\tilde{a}_{3} \tilde{x}+\tilde{a}_{6} \tilde{y}-c_{14} & \tilde{x}\left(\tilde{x}+a_{8}\right)+c_{13} \\
-\tilde{x} & -\tilde{y} \\
\tilde{z} & b_{12}\left(\tilde{x}+a_{8}\right) \\
b_{12} \tilde{a}_{6} & \tilde{z}+b_{12} \tilde{a}_{3}
\end{array}\right)
$$

where again $\tilde{x}=x+a_{2} w, \tilde{y}=y+a_{1} w, \tilde{z}=z+a_{11} w+a_{9} x+a_{10} y, \tilde{a}_{3}=a_{3} w+a_{4} y+a_{5}$ and $\tilde{a}_{6}=a_{6} w+a_{7} y$.

The map $M_{\mathbb{P}^{3}}^{3 t+1} \longrightarrow \mathrm{Hilb}_{\mathbb{P}^{3}}^{3 t+1}$ is given by the identity map on the variables $a_{1}, \ldots, a_{11}, b_{12}, c_{13}, c_{14}$.

Proof. Infinitesimal deformations of the matrices $A$ and $B$ can be found by direct computation, see also [FT04, Section 7]. Then one has to try to lift the equation $A B=0$. The result is as stated. One has

$$
\widetilde{A B}=\left(\begin{array}{cc}
-c_{13} b_{12} \tilde{a}_{6} & c_{14} b_{12}\left(\tilde{x}+a_{8}\right)-c_{13} b_{12} \tilde{a}_{3} \\
c_{14} b_{12} & -c_{13} b_{12}
\end{array}\right)
$$

Therefore the obstruction to lift the equation is given by the ideal $J=\left(b_{12} c_{13}, b_{12} c_{14}\right)$.

On the component $c_{13}=c_{14}=0$ the map to the Hilbert scheme is induced by taking the $0^{\text {th }}$ Fitting ideal. This does not work on the component $b_{12}=0$. Freiermuth and Trautmann show that the larger component is isomorphic to the relative universal cubic in the bundle of hyperplanes of $\mathbb{P}^{3}$ [FT04, §6.5]. The map to the Hilbert scheme associates to a pair consisting of a cubic in a hyperplane and a point on it the scheme consisting of this cubic with an embedded point at this point.

Remark B.2. The matrix $\widetilde{A}$, restricted to the component $c_{13}=c_{14}=0$, gives a presentation of $i_{R *} \mathscr{O}_{X_{R}}$ with $\left(X_{R}, i_{R}\right)$ the universal family of Lemma 2.13. To see this we use a coordinate transformation from the 
coordinates in the Lemma. We set $\tilde{x}=x+a_{2} w, \tilde{y}=y+a_{1} w, \tilde{z}=z-b_{11} w-b_{10} y-b_{9} x, \tilde{a}_{3}=a_{3} w+a_{4} y+a_{5} x$ and $\tilde{a}_{6}=a_{6} w+a_{7} y$. We furthermore put $\tilde{a}_{8}=a_{8}-a_{2}$. Then the curve becomes in the chart $\{w=1\}$

$$
\left(\begin{array}{ccc}
\tilde{x} & \tilde{a}_{6} & u \\
\tilde{y} & u+\tilde{a}_{3} & \tilde{x}+\tilde{a}_{8}
\end{array}\right)
$$

and the morphism $i_{R}^{*}$ is $(\tilde{x}, \tilde{y}, \tilde{z}) \mapsto\left(\tilde{x}, \tilde{y}, b_{12} u\right)$.

\section{References}

[Art76] M. Artin, Lectures on deformations of singularities, Lectures on Mathematics and Physics, vol. 54, Tata Institute of Fundamental Research, Bombay, 1976.

[Buc81] R.-O. Buchweitz, Contributions à la théorie des singularités : Déformations de Diagrammes, Déploiements et Singularités très rigides, Liaison algébrique, 1981, Thesis, École Polytechnique. Available from http://hdl. handle.net/1807/16684.

[Cat84] F. Catanese, Commutative algebra methods and equations of regular surfaces, Algebraic geometry, Bucharest 1982 (Bucharest, 1982), Lecture Notes in Math., vol. 1056, Springer, Berlin, 1984, pp. $68-111$.

[CCN11] D. Chen, I. Coskun, and S. Nollet, Hilbert scheme of a pair of codimension two linear subspaces, Comm. Algebra 39 (2011), no. 8, 3021-3043.

[Che08] D. Chen, Mori's program for the Kontsevich moduli space $\overline{\mathscr{M}}_{0,0}\left(\mathbb{P}^{3}, 3\right)$, Int. Math. Res. Not. (2008), article id. rnn067.

[CK11] K. Chung and Y.-H. Kiem, Hilbert scheme of rational cubic curves via stable maps, Amer. J. Math. 133 (2011), no. 3, 797-834.

[dJvS90] T. de Jong and D. van Straten, Deformations of the normalization of hypersurfaces, Math. Ann. 288 (1990), no. 3, 527-547.

[Eis95] D. Eisenbud, Commutative algebra. With a view toward algebraic geometry, Graduate Texts in Mathematics, vol. 150, Springer-Verlag, New York, 1995.

[EPS87] G. Ellingsrud, R. Piene, and S. A. Strømme, On the variety of nets of quadrics defining twisted cubics, in: Space curves (Rocca di Papa, 1985), Lecture Notes in Math., vol. 1266, Springer, Berlin, 1987, pp. 84-96.

[FT04] H. G. Freiermuth and G. Trautmann, On the moduli scheme of stable sheaves supported on cubic space curves, Amer. J. Math. 126 (2004), no. 2, 363-393.

[Got08] G. Gotzmann, The irreducible components of $\mathrm{Hilb}^{4 n}\left(\mathbf{P}^{3}\right)$, Preprint, arXiv:0811.3160, 2008.

[Gro63] A. Grothendieck, Éléments de géométrie algébrique. III. Étude cohomologique des faisceaux cohérents, Seconde partie, Inst. Hautes Études Sci. Publ. Math. (1963), no. 17, 5-91.

[Har82] J. Harris, Curves in projective space, Séminaire de Mathématiques Supérieures [Seminar on Higher Mathematics], vol. 85, Presses de l’Université de Montréal, Montreal, Que., 1982.

[Har94] R. Hartshorne, The genus of space curves, Ann. Univ. Ferrara Sez. VII (N.S.) 40 (1994), 207-223 (1996).

[Hei14] K. Heinrich, The space of Cohen-Macaulay curves and related topics, 2014, Thesis, KTH, URN: urn:nbn:se:kth:diva-143966. 
[Høn05] M. O. Hønsen, Compactifying locally Cohen-Macaulay projective curves, 2005, Thesis, KTH, URN: urn:nbn:se:kth:diva-470.

[IIl72] L. Illusie, Complexe cotangent et déformations. II, Lecture Notes in Mathematics, Vol. 283, SpringerVerlag, Berlin-New York, 1972.

[KLU92] S. Kleiman, J. Lipman, and B. Ulrich, The source double-point cycle of a finite map of codimension one, in: Complex projective geometry (Trieste, 1989/Bergen, 1989), London Math. Soc. Lecture Note Ser., vol. 179, Cambridge Univ. Press, Cambridge, 1992, pp. 199-212.

[Kon95] M. Kontsevich, Enumeration of rational curves via torus actions, in: The moduli space of curves (Texel Island, 1994), Progr. Math., vol. 129, Birkhäuser Boston, Boston, MA, 1995, pp. 335-368.

[LR11] P. Lella and M. Roggero, Rational components of Hilbert schemes, Rend. Semin. Mat. Univ. Padova 126 (2011), 11-45.

[Mum88] D. Mumford, The red book of varieties and schemes, Lecture Notes in Mathematics, vol. 1358, Springer-Verlag, Berlin, 1988.

[Nor76] D. G. Northcott, Finite free resolutions, Cambridge University Press, Cambridge, 1976, Cambridge Tracts in Mathematics, No. 71.

[PS85] R. Piene and M. Schlessinger, On the Hilbert scheme compactification of the space of twisted cubics, Amer. J. Math. 107 (1985), no. 4, 761-774.

[Sch73] M. Schaps, Déformations non singulières de courbes gauches, in: Singularités à Cargèse (Rencontre sur les singularités en géométrie analytique, Cargèse, 1972), 1973, Astérisque, nos. 7 and 8, pp. 121128.

[Sch99] E. Schlesinger, On the spectrum of certain subschemes of $\mathbf{P}^{N}$, J. Pure Appl. Algebra 136 (1999), no. 3, 267-283.

[Ste95] J. Stevens, Computing versal deformations, Experiment. Math. 4 (1995), no. 2, 129-144.

[Tei77] B. Teissier, The hunting of invariants in the geometry of discriminants, in: Real and complex singularities (Proc. Ninth Nordic Summer School/NAVF Sympos. Math., Oslo, 1976), Sijthoff and Noordhoff, Alphen aan den Rijn, 1977, pp. 565-678.

[VA92] I. Vainsencher and D. Avritzer, Compactifying the space of elliptic quartic curves, in: Complex projective geometry (Trieste, 1989/Bergen, 1989), London Math. Soc. Lecture Note Ser., vol. 179, Cambridge Univ. Press, Cambridge, 1992, pp. 47-58.

[VX02] I. Vainsencher and F. Xavier, A compactification of the space of twisted cubics, Math. Scand. 91 (2002), no. 2, 221-243. 